\title{
THE GRIFFITHS GROUP OF A GENERAL CALABI-YAU THREEFOLD IS NOT FINITELY GENERATED
}

\section{CLAIRE VOISIN}

1. Introduction. If $X$ is a Kähler variety, the intermediate Jacobian $J^{2 k-1}(X)$ is defined as the complex torus

$$
J^{2 k-1}(X)=H^{2 k-1}(X, \mathbb{C}) / F^{k} H^{2 k-1}(X) \oplus H^{2 k-1}(X, \mathbb{Z}),
$$

where $F^{k} H^{2 k-1}(X)$ is the set of classes representable by a closed form in $F^{k} A^{2 k-1}(X)$, that is, which is locally of the form $\sum_{I, J} \alpha_{I, J} d z_{I} \wedge d \bar{z}_{J}$, with $|I|+|J|=2 k-1$ and $|I| \geq k$.

Griffiths [9] has defined the Abel-Jacobi map

$$
\Phi_{X}^{k}: \mathscr{E}_{\mathrm{hom}}^{k}(X) \longrightarrow J^{2 k-1}(X),
$$

where $\mathscr{L}_{\text {hom }}^{k}(X)$ is the group of codimension $k$ algebraic cycles homologous to zero on $X$. Using the identification

$$
J^{2 k-1}(X)=\frac{\left(F^{n-k+1} H^{2 n-2 k+1}(X)\right)^{*}}{H_{2 n-2 k+1}(X, \mathbb{Z})}, \quad n=\operatorname{dim} X
$$

given by Poincaré duality, $\Phi_{X}^{k}$ associates to the cycle $Z=\partial \Gamma$, where $\Gamma$ is a real chain of dimension $2 n-2 k+1$ well defined up to a $2 n-2 k+1$-cycle, the element

$$
\int_{\Gamma} \in\left(F^{n-k+1} H^{2 n-2 k+1}(X)\right)^{*} / H_{2 n-2 k+1}(X, \mathbb{Z}),
$$

which is well defined using the isomorphism

$$
F^{n-k+1} H^{2 n-2 k+1}(X) \cong \frac{F^{n-k+1} A^{2 n-2 k+1}(X)^{c}}{d F^{n-k+1} A^{2 n-2 k}(X)} .
$$

If $\left(Z_{t}\right)_{t \in C}$ is a flat family of codimension $k$ algebraic cycles on $X$ parametrized by a smooth irreducible curve $C$, the map $t \mapsto \Phi_{X}^{k}\left(Z_{t}-Z_{0}\right)$ factors through a homomorphism from the Jacobian $J(C)$ to $J^{2 k-1}(X)$, and one can show that the image of this morphism is a complex subtorus of $J^{2 k-1}(X)$ whose tangent space is contained in $H^{k-1, k}(X) \subset H^{2 k-1}(X, \mathbb{C}) / F^{k} H^{2 k-1}(X)$. Defining the subgroup

Received 9 March 1999.

1991 Mathematics Subject Classification. Primary 14C25, 14C30, 14M99, 32 J25.

Author's work partially supported by the project Algebraic Geometry in Europe. 
$\mathscr{E}_{\mathrm{alg}}^{k}(X) \subset \mathscr{L}_{\mathrm{hom}}^{k}(X)$ of cycles algebraically equivalent to zero as the subgroup generated by the cycles $Z_{t}-Z_{0}$ for any family as above and defining the Griffiths group $\operatorname{Griff}^{k}(X)$ as the quotient $\mathscr{L}_{\text {hom }}^{k}(X) / \mathscr{L}_{\text {alg }}^{k}(X)$, it follows that the Abel-Jacobi map induces a morphism

$$
\Phi_{X}^{k}: \operatorname{Griff}^{k}(X) \longrightarrow J^{2 k-1}(X)_{\mathrm{tr}},
$$

where $J^{2 k-1}(X)_{\text {tr }}$ is the quotient of $J^{2 k-1}(X)$ by its maximal subtorus having its tangent space contained in $H^{k-1, k}(X)$.

In this paper, we are mainly interested in the case where $n=3, k=2$. We use then the notation $J(X), \Phi_{X}$. In [10], Griffiths proved the following theorem.

THEOREM 1. If $X$ is a general quintic threefold and $Z$ is the difference of two distinct lines in $X, \Phi_{X}(Z)$ is not a torsion point in $J(X)$. Furthermore, $J(X)_{\operatorname{tr}}=J(X)$.

From this it follows that $\operatorname{Griff}(X)$ contains nontorsion elements.

In [3] Clemens, using the countably many isolated rational curves in $X$, proved the following theorem.

Theorem 2. If $X$ is a general quintic threefold, $\operatorname{Im} \Phi_{X} \otimes \mathbb{Q}$ is not a finitedimensional $\mathbb{Q}$-vector space. In particular, $\operatorname{Griff}(X) \otimes \mathbb{Q}$ is not a finite-dimensional $\mathbb{Q}$-vector space.

Clemens's theorem has been extended to complete intersections by Paranjape [15] and to Abelian threefolds by Nori [14]. (In the last case, $J(X)_{\text {tr }}$ is different from $J(X)$, and one considers the Abel-Jacobi map with value in $J(X)_{\mathrm{tr}}$.) Notice that it is conjectured (see [13]) that for codimension-two cycles, the Abel-Jacobi map $\Phi_{X}^{2}$ : Griff $(X) \rightarrow J(X)_{\text {tr }}$ is injective, so both statements should be equivalent.

More recently, Nori [13] proved that there may exist nontorsion cycles in $\operatorname{Griff}^{k}(X)$ for any $k \geq 3$ (so $X$ has to be of dimension at least 4), which are annihilated by the Abel-Jacobi map. Combining Nori's ideas and the study of the Abel-Jacobi map for the general cubic sevenfold in $\mathbb{P}^{8}$, Albano and Collino [1] even proved that for $k \geq 3$ the kernel of the Abel-Jacobi map $\Phi_{X}^{k}: \operatorname{Griff}^{k}(X) \rightarrow J^{2 k-1}(X)_{\text {tr }}$ may be nonfinitely generated.

In this paper, we consider another kind of generalization of the Clemens theorem: Instead of a quintic threefold, we consider a Calabi-Yau threefold $X$; that is, $X$ is a Kähler threefold with trivial canonical bundle such that $H^{2}\left(\mathbb{O}_{X}\right)=0$ (so, in particular, $X$ is projective). For such $X$ it is well known that the local moduli space of $X$ is smooth of dimension $\operatorname{dim} H^{1}\left(T_{X}\right)=\operatorname{dim} H^{1,2}(X)$. In [17] we proved the following.

Theorem 3. Let $X$ be a Calabi-Yau threefold. If $h^{1}\left(T_{X}\right) \neq 0$, the general deformation $X_{t}$ of $X$ satisfies that the Abel-Jacobi map

$$
\Phi_{X_{t}}: \mathscr{L}^{2}\left(X_{t}\right) \longrightarrow J^{2}\left(X_{t}\right)
$$

of $X_{t}$ is nontrivial, even modulo torsion. 
It is easy to check that $J\left(X_{t}\right)_{\operatorname{tr}}=J\left(X_{t}\right)$ for a general point $t$, so the theorem implies that $\operatorname{Griff}\left(X_{t}\right)$ contains nontorsion elements. We prove in this paper the following result.

Theorem 4. Let $X$ be a Calabi-Yau threefold. If $h^{1}\left(T_{X}\right) \neq 0$, the general deformation $X_{t}$ of $X$ has the property that the Abel-Jacobi map

$$
\Phi_{X_{t}}: \mathscr{L}^{2}\left(X_{t}\right) \longrightarrow J\left(X_{t}\right)
$$

is such that $\operatorname{Im} \Phi_{X_{t}} \otimes \mathbb{Q}$ is an infinite-dimensional $\mathbb{Q}$-vector space. In particular, $\operatorname{Griff}\left(X_{t}\right) \otimes \mathbb{Q}$ is an infinite-dimensional $\mathbb{Q}$-vector space.

The one-cycles in $X_{t}$ we use to prove this result are the same as in [18]. Namely, we consider for $\left|L_{t}\right|$ a sufficiently ample linear system on $X_{t}$, the surfaces $S \in\left|L_{t}\right|$, $S \stackrel{j_{S}}{\longrightarrow} X_{t}$ having a class $\lambda \in \operatorname{Ker}\left(j_{S_{*}}: H^{2}(S, \mathbb{Z}) \rightarrow H^{4}\left(X_{t}, \mathbb{Z}\right)\right)$, which is in $F^{1} H^{2}(S)$; that is, $\lambda$ is algebraic, $\lambda=c_{1}\left(D_{\lambda}\right)$ for some divisor $D_{\lambda}$ on $S$, by the Lefschetz theorem on $(1,1)$-classes.

It was proved in [17] that there are countably many isolated such surfaces in $X_{t}$, and the countably many corresponding one-cycles $Z_{\lambda}=j_{S_{*}}\left(D_{\lambda}\right)$ homologous to zero in $X_{t}$ were proved in [18] to generate a nontorsion subgroup of $J\left(X_{t}\right)$ by the Abel-Jacobi map. We were unable to show, however, that this subgroup is nonfinitely generated.

The method we use is in some sense related to a suggestion of Clemens in [4]. He suggested that a proof of the nonfinite generation of the Griffiths group of the general quintic threefold could be obtained by studying the ramification loci of the various generically finite coverings $\pi_{d}: M_{d} \rightarrow \mathcal{M}$, where $\mathcal{M}$ is the moduli space for the quintic threefold and $M_{d}$ parametrizes a quintic threefold $X$ and a degree $d$ isolated rational curve $C$ in it. Along the ramification divisor of $\pi_{d}$, the curve $C \subset X$ has an infinitesimal deformation $\eta$ in $X$, and there is a corresponding element $\Phi_{X *}(\eta) \in H^{1,2}(X)$, which is the differential of $\Phi_{X}$ applied to the deformation $\eta$ of the corresponding cycle in $X$.

However, another important ingredient is the complexified Abel-Jacobi map; we use the complexified infinitesimal Abel-Jacobi map to prove Theorem 4. The "complexified" objects we study are the following: If $S \stackrel{j_{S}}{\longrightarrow} X$, and $\lambda \in \operatorname{Ker}\left(j_{S_{*}}: H^{2}(S, \mathbb{C}) \rightarrow\right.$ $\left.H^{4}\left(X_{t}, \mathbb{C}\right)\right)$, we define $U_{\lambda}$ as the set of deformations $\left(X_{t}, S_{t}\right)$ of the pair $(X, S)$ such that the fixed class $\lambda_{t} \in H^{2}\left(S_{t}, \mathbb{C}\right) \cong H^{2}(S, \mathbb{C})$ belongs to $F^{1} H^{2}\left(S_{t}\right)$. It turns out that when $X$ is a Calabi-Yau threefold and $\mathcal{M}$ is its local moduli space, most varieties $U_{\lambda}$ are generically finite covers of $\mathcal{M}$ (by the map $\left.\left(X_{t}, S_{t}\right) \mapsto X_{t}\right)$. A point $\left(X_{t}, S_{t}\right)$ of ramification of this map then corresponds to a surface $S_{t} \subset X_{t}$ that admits an infinitesimal deformation $\eta$ such that $\lambda_{t} \in F^{1} H^{2}\left(S_{t}\right)$ remains (infinitesimally) in $F^{1} H^{2}\left(S_{t}^{\eta}\right)$. There is then an associated complexified infinitesimal Abel-Jacobi invariant $\Phi_{X_{t *}}(\eta) \in H^{1,2}\left(X_{t}\right)$. Notice that if $\lambda$ is integral, it is the class of a divisor in $S_{t}$ and we get the same invariant as above. 
In Section 2, we introduce various Hodge theoretic objects and study the varieties $U_{\lambda}$ defined above. We also define the complexified Abel-Jacobi map and "compute" its differential.

In Section 3, we give a very simple infinitesimal criterion, which implies that the infinitesimal invariants above are nonzero and that if the image of the Abel-Jacobi map of $X_{t}$ were finitely generated, these infinitesimal invariants would vanish. It follows that if this criterion is satisfied, then Theorem 4 is true.

This infinitesimal criterion concerns the infinitesimal variation of Hodge structure of a generic sufficiently ample surface $S \subset X$. In Section 4, we check this criterion, which reduces (see [7]) to the study of Jacobian rings, that is, quotients of rings of functions by Jacobian ideals, generated by the derivatives of the defining equation of the surface along vector fields.

2. Noether-Lefschetz loci and infinitesimal Abel-Jacobi map. Part of the material in this section works in the general situation of a family of smooth surfaces $\mathscr{S} \rightarrow B$ contained in a family of smooth threefolds $\mathscr{X} \rightarrow B$; however, we restrict the discussion to the following situation: $\mathscr{X} \stackrel{\pi}{\rightarrow} B$ is the local universal family of deformations of a Calabi-Yau threefold $X . B$ is a smooth ball, which can be assumed to be as small as we want. We have $\operatorname{dim} B=\operatorname{dim} H^{1}\left(T_{X}\right)$. Now let $L$ be an ample line bundle on $X$; since $H^{1}\left(\mathscr{O}_{X}\right)=H^{2}\left(\mathscr{O}_{X}\right)=0$ and $H^{i}(L)=0, i>0$, by Kodaira vanishing and $K_{X}$ trivial, $L$ extends uniquely to a line bundle $\mathscr{L}$ on $\mathscr{X}$, and $\operatorname{dim} H^{0}\left(X_{t}, L_{t}\right)=\operatorname{dim} H^{0}(X, L)$ for any $t \in B$. Then $\mathbb{P}\left(R^{0} \pi_{*} \mathscr{L}\right) \stackrel{p}{\rightarrow} B$ is smooth over $B$, and we denote by $U \subset \mathbb{P}\left(R^{0} \pi_{*} \mathscr{L}\right)$ the open set parametrizing smooth surfaces. Let then $\mathscr{S} \stackrel{\pi_{S}}{\longrightarrow} U$ be the universal family, $\mathscr{X}_{U} \stackrel{\pi_{X}}{\longrightarrow} U$ be the pullback to $U$ of the family $\mathscr{X} \stackrel{\pi}{\rightarrow} B$, and $j: \mathscr{S} \hookrightarrow \mathscr{X}_{U}$ be the natural inclusion. First we have the following lemma.

LemMa 1. For sufficiently ample $L$, the tangent space $T_{U, t}$ at a point $t$ identifies to $H^{1}\left(T_{S_{t}}\right)$ by the Kodaira-Spencer map. It is also isomorphic to $H^{1}\left(T_{X_{t}}^{S_{t}}\right)$ by the Kodaira-Spencer map for pairs, where $T_{X_{t}}^{S_{t}}$ is the kernel of the natural map

$$
T_{X_{t}}^{S_{t}} \longrightarrow N_{S_{t} / X_{t}}
$$

Proof. We have the exact sequence

$$
0 \longrightarrow T_{X_{t}}\left(-L_{t}\right) \longrightarrow T_{X_{t}}^{S_{t}} \longrightarrow T_{S_{t}} \longrightarrow 0,
$$

which induces the natural map

$$
H^{1}\left(T_{X_{t}}^{S_{t}}\right) \longrightarrow H^{1}\left(T_{S_{t}}\right)
$$

from the deformations of the pair to the deformations of the surface. So by Serre vanishing, the map above is an isomorphism for sufficiently ample $L$. 
Next we have the exact sequence

$$
0 \longrightarrow H^{0}\left(L_{t \mid S_{t}}\right) \longrightarrow T_{U, t} \stackrel{p_{*}}{\longrightarrow} T_{B, p(t)} \longrightarrow 0
$$

and the exact sequence defining $T_{X_{t}}^{S_{t}}$,

$$
0 \longrightarrow T_{X_{t}}^{S_{t}} \longrightarrow T_{X_{t}} \rightarrow L_{t \mid S_{t}} \longrightarrow 0
$$

which induces the exact sequence

$$
0 \longrightarrow H^{0}\left(L_{t \mid S_{t}}\right) \longrightarrow H^{1}\left(T_{X_{t}}^{S_{t}}\right) \longrightarrow H^{1}\left(T_{X_{t}}\right) \longrightarrow 0
$$

since $H^{0}\left(T_{X_{t}}\right)=0$ and $H^{1}\left(L_{t \mid S_{t}}\right)=0$. Finally, the Kodaira-Spencer map $T_{U, t} \rightarrow$ $H^{1}\left(T_{X_{t}}^{S_{t}}\right)$ fits into the commutative diagram

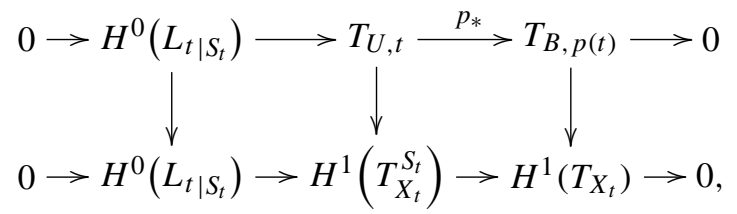

where the first and last vertical maps are the identity. It follows immediately that the middle map is an isomorphism.

We have on $U$ the primitive variation of Hodge structure of the family of surfaces $\mathscr{S}$ : namely, let

$$
H_{\mathbb{Z}}^{2}:=\operatorname{Ker}\left(R^{2} \pi_{S *} \mathbb{Z} \stackrel{j_{*}}{\longrightarrow} R^{4} \pi_{X *} \mathbb{Z}\right)
$$

be the local system whose fiber at $t$ is

$$
H^{2}\left(S_{t}, \mathbb{Z}\right)_{0}:=\operatorname{Ker}\left(H^{2}\left(S_{t}, \mathbb{Z}\right) \stackrel{j_{t *}}{\longrightarrow} H^{4}\left(X_{t}, \mathbb{Z}\right)\right) .
$$

Let $\mathscr{H}^{2}:=H_{\mathbb{Z}}^{2} \otimes \mathcal{O}_{U}$, with its Gauss-Manin connection $\nabla^{S}: \mathscr{H}^{2} \rightarrow \mathscr{H}^{2} \otimes \Omega_{U}$, whose local system of flat sections is $H_{\mathbb{C}}^{2}=H_{\mathbb{Z}}^{2} \otimes \mathbb{C}$. Let $F^{i} \mathcal{H}^{2}, 0 \leq i \leq 2$ be the Hodge bundles, with fiber

$$
F^{i} \mathscr{H}_{t}^{2}=F^{i} H^{2}\left(S_{t}\right) \cap \operatorname{Ker} j_{t *}, \quad F^{i} H^{2}\left(S_{t}\right)=\oplus_{p \geq i} H^{p, 2-p}\left(S_{t}\right)
$$

and associated quotients $\mathscr{H}^{i, 2-i}=F^{i} \mathscr{H}^{2} / F^{i+1} \mathscr{H}^{2}$. By transversality, we have

$$
\nabla^{S} F^{i} \mathscr{H}^{2} \subset F^{i-1} \mathscr{H}^{2} \otimes \Omega_{U} .
$$

We denote by

$$
\bar{\nabla}^{S}: \mathscr{H}^{i, 2-i} \longrightarrow \mathscr{H}^{i-1,3-i} \otimes \Omega_{U}
$$


the $\mathfrak{O}_{U}$-linear map deduced from $\nabla^{S}$ by transversality, so that $\bar{\nabla}^{S}$ fits into the commutative diagram

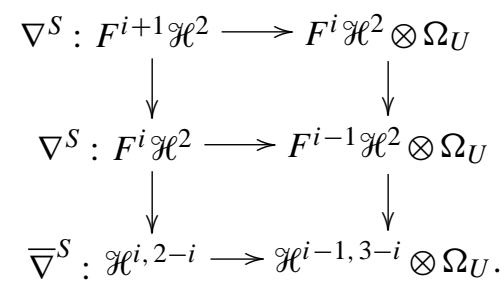

For $\lambda \in H^{i, j}\left(S_{v}\right)_{0}$ we then have $\bar{\nabla}^{S}(\lambda) \in \operatorname{Hom}\left(T_{U, v}, H^{i-1, j+1}\left(S_{v}\right)_{0}\right)$. For $\eta \in T_{U, v}$, we denote by $\bar{\nabla}_{\eta}^{S}$ the induced map $H^{i, j}\left(S_{v}\right)_{0} \rightarrow H^{i-1, j+1}\left(S_{v}\right)_{0}$.

Let $V$ be a simply connected open subset of $U$. Then the local system $H_{\mathbb{C}}^{2}$ is trivial on $V$, so that if $v_{0} \in V$ and $\lambda \in H^{2}\left(S_{v_{0}}, \mathbb{C}\right)_{0}$, we can view $\lambda$ as a section of $H_{\mathbb{C}}^{2}$ on $V$. We then define the component of the Noether-Lefschetz locus determined by $\lambda$ as

$$
V_{\lambda}=\left\{t \in V, \lambda_{t} \in F^{1} H^{2}\left(S_{t}\right)_{0}\right\} .
$$

$V_{\lambda}$ is an analytic subvariety of $V$, defined by the vanishing of the projection in $\mathscr{H}^{0,2}$ of the flat, hence holomorphic, section $\lambda \in \mathscr{H}^{2}$. If $t \in V_{\lambda}, \lambda_{t} \in F^{1} H^{2}\left(S_{t}\right)_{0}$ and hence has a projection $\lambda_{t}^{1,1} \in H^{1,1}\left(S_{t}\right)_{0}=\mathscr{H}_{t}^{1,1}$. Then the next lemma follows from the definition of $\bar{\nabla}^{S}$.

Lemma 2. The Zariski tangent space to $V_{\lambda}$ at $t$ is equal to $\operatorname{Ker} \bar{\nabla}^{S}\left(\lambda_{t}^{1,1}\right)$, where $\bar{\nabla}^{S}\left(\lambda_{t}^{1,1}\right) \in \operatorname{Hom}\left(T_{V, t}, H^{0,2}\left(S_{t}\right)\right)$.

Note that usually the terminology of the Noether-Lefschetz locus is reserved to the case where $\lambda$ is rational. In this case, by the Lefschetz theorem on $(1,1)$-classes, $V_{\lambda}$ is the set of points $v \in V$ where the class $\lambda_{v}$ is algebraic; that is, any multiple $m_{\lambda} \lambda_{v}$ that is an integral class is the class $\left[D_{\lambda, v}\right]$ of a divisor on $S_{v}$. Then since $j_{v *} \lambda_{v}=0$, $j_{v *}\left(D_{\lambda, v}\right)$ is a one-cycle homologous to zero in $X_{v}$.

We have the following convenient interpretation of $V_{\lambda}$ : Let $v_{0}$ be any point of $V$; then $H_{\mathbb{C}}^{2} \cong V \times H^{2}\left(S_{v_{0}}, \mathbb{C}\right)_{0}$. Viewing $F^{1} \mathscr{H}^{2}, \mathscr{H}^{2}$ as vector bundles, we have a map

$$
\phi: F^{1} \mathscr{H}^{2} \longrightarrow H^{2}\left(S_{v_{0}}, \mathbb{C}\right)_{0}
$$

obtained as the composition of the inclusion $F^{1} \mathscr{H}^{2} \subset \mathscr{H}^{2}$, the isomorphism $\mathscr{H}^{2} \cong$ $H^{2}\left(S_{v_{0}}, \mathbb{C}\right)_{0} \times V$ given by the trivialization of $H_{\mathbb{C}}^{2}$, and the first projection. Then we have that $V_{\lambda}$ is naturally isomorphic to $\phi^{-1}\left(\lambda_{v_{0}}\right)$. Indeed, by definition, $V_{\lambda} \times \lambda_{v_{0}} \subset$ $V \times H^{2}\left(S_{v_{0}}, \mathbb{C}\right)_{0} \cong \mathscr{H}^{2}$ is the scheme-theoretic intersection of $V_{\lambda} \times \lambda_{v_{0}}$ and $F^{1} \mathscr{H}^{2}$ in $\mathscr{H}^{2}$; but this is also the definition of the fiber $\phi^{-1}\left(\lambda_{v_{0}}\right)$.

In other words, the flat section $\lambda$ restricted to $V_{\lambda}$, which is in $F^{1} \mathscr{H}_{\mid V_{\lambda}}^{2}$, gives the reverse isomorphism $V_{\lambda} \rightarrow \phi^{-1}\left(\lambda_{v_{0}}\right)$. We abuse notation in Section 3 and view, by this isomorphism, $V_{\lambda}$ as a subvariety of $F^{1} \mathscr{H}_{\mid V}^{2}$. 
We denote by

$$
\lambda^{1,1} \in \mathscr{H}_{\mid V_{\lambda}}^{1,1}
$$

the projection of the section $\lambda \in F^{1} \mathscr{H}_{\mid V}^{2}$. Now if $v \in V$ and $\lambda^{1,1} \in H^{1,1}\left(S_{v}\right)_{0}$, let $\lambda_{1}, \lambda_{2} \in F^{1} H^{2}\left(S_{v}\right)_{0}$ be two liftings of $\lambda^{1,1}$, so that $\lambda_{1}=\lambda_{2}+\eta$, for some $\eta \in$ $H^{2,0}\left(S_{v}\right)$. By Lemma 2 the tangent spaces to $V_{\lambda_{i}}$ at $v$ coincide, and the two sections $\lambda_{i}^{1,1}$, which are defined on the first-order neighbourhood $V_{\lambda}^{\epsilon}$ of $v$ in $V_{\lambda_{i}}$ (where $i=1$ or 2) are equal at $v$. However, their derivatives do not coincide. In fact, we have the next lemma.

Lemma 3. The derivative at $v$ of the section $\lambda_{1}^{1,1}-\lambda_{2}^{1,1}$ of $\mathscr{H}_{\mid V_{\lambda}^{\epsilon}}^{1,1}$ (which vanishes at $v$ ), is equal to $-\bar{\nabla}(\eta)_{\mid T_{V_{\lambda}, v}}: T_{V_{\lambda}, v} \rightarrow H^{1,1}\left(S_{v}\right)_{0}$.

Proof. Let $h \in T_{V_{\lambda}^{\epsilon}, v}$ and let $Z_{h}$ be the scheme of length two supported on $v$ with tangent vector $h$. Then the section $\lambda_{1}^{h}=\lambda_{1 \mid Z_{h}}$ of $F^{1} \mathscr{H}^{2}$ is the flat section that extends $\lambda_{1} \in F^{1} H^{2}\left(S_{v}\right)_{0}$ and that remains in $F^{1} \mathscr{H}^{2}$. Now, $\eta$ being given above, let

$$
\mu_{2}^{h}:=\lambda_{1}^{h}+\epsilon \nabla_{h}^{S}(\tilde{\eta})-\tilde{\eta},
$$

where $\tilde{\eta}$ is a section of $F^{2} \mathscr{H}^{2}$ on $Z_{h}$ extending $\eta$. Then clearly $\mu_{2}^{h}$ is flat and its value at $v$ is equal to $\lambda_{2}$. Furthermore, $\mu_{2}^{h}$ is a section of $F^{1} \mathscr{H}^{2}$ on $Z_{h}$ by transversality. It follows that $\lambda_{2}^{h}=\mu_{2}^{h}$. Hence,

$$
\lambda_{1}^{h}-\lambda_{2}^{h}=-\epsilon \nabla_{h}(\tilde{\eta})+\tilde{\eta},
$$

so that by projecting to $\mathscr{H}^{1,1}$ and using the definition of $\bar{\nabla}^{S}$, we get

$$
\left(\lambda_{1}^{h}\right)^{1,1}-\left(\lambda_{2}^{h}\right)^{1,1}=-\epsilon \bar{\nabla}^{S}(\eta)(h),
$$

which proves the lemma.

We now turn to the generalized Abel-Jacobi map and its infinitesimal version. For $u \in U$, let $Y_{u}=X_{u}-S_{u}$. We have an exact sequence

$$
0 \longrightarrow H^{3}\left(X_{u}\right) \longrightarrow H^{3}\left(Y_{u}\right) \stackrel{\text { Res }}{\longrightarrow} H^{2}\left(S_{u}\right)_{0} \longrightarrow 0
$$

of cohomology groups with integral coefficients, and $H^{3}\left(Y_{u}, \mathbb{C}\right)$ carries a mixed Hodge structure compatible with the Hodge structures on $H^{3}\left(X_{u}\right)$ and $H^{2}\left(S_{u}\right)_{0}$. Namely, we have a decreasing filtration $F^{i} H^{3}\left(Y_{u}\right), 0 \leq i \leq 3$, such that

$$
F^{i} H^{3}\left(Y_{u}\right) \cap H^{3}\left(X_{u}\right)=F^{i} H^{3}\left(X_{u}\right), \quad \operatorname{Res}\left(F^{i} H^{3}\left(Y_{u}\right)\right)=F^{i-1} H^{2}\left(S_{u}\right)_{0},
$$

where $F^{i} H^{3}\left(X_{u}\right)=\oplus_{p \geq i} H^{p, 3-p}\left(X_{u}\right)$ is the Hodge filtration of $X_{u}$. 
Working in families, we get the local system

$$
H_{Y, \mathbb{Z}}^{3}=R^{3} \pi_{Y *} \mathbb{Z}
$$

where $\pi_{Y}=\pi_{X \mid \mathscr{y}}, \mathscr{Y}=\mathscr{X}_{U}-\mathscr{Y}$. We then define the associated Hodge bundles $\mathscr{H}_{Y}^{3}$ by tensorizing the local system with $\mathscr{O}_{U}$. We denote by $\nabla^{Y}$ the Gauss-Manin connection on $\mathscr{H}_{Y}^{3}$. This bundle is equipped with the Hodge filtration by holomorphic subbundles $F^{i} \mathscr{H}_{Y}^{3}$, which satisfy Griffiths transversality

$$
\nabla^{Y} F^{i} \mathscr{H}_{Y}^{3} \subset F^{i-1} \mathscr{H}_{Y}^{3} \otimes \Omega_{U}
$$

We denote by $H_{\mathbb{Z}}^{3}, \mathscr{H}^{3}, F^{i} \mathscr{H}^{3}$, and $\nabla^{X}$ the analogous objects on $B$ that describe the variation of Hodge structure of the family $\pi: \mathscr{L} \rightarrow B$; that is, $H_{\mathbb{Z}}^{3}=R^{3} \pi_{*} \mathbb{Z}, F^{i} \mathscr{H}^{3} \subset$ $\mathscr{H}^{3}$ with $\mathscr{H}^{3}=H_{\mathbb{Z}}^{3} \otimes \mathcal{O}_{B}$, and $\nabla^{X}: \mathscr{H}^{3} \rightarrow \mathscr{H}^{3} \otimes \Omega_{B}$ with

$$
\nabla^{X} F^{i} \mathscr{H}^{3} \subset F^{i-1} \mathscr{H}^{3} \otimes \Omega_{B} .
$$

We then have an exact sequence of variation of mixed Hodge structures

$$
0 \longrightarrow p^{*} H_{\mathbb{Z}}^{3} \longrightarrow H_{Y, \mathbb{Z}}^{3} \longrightarrow H_{\mathbb{Z}}^{2} \longrightarrow 0 .
$$

On our open set $V$, let us choose a splitting $r_{\mathbb{Z}}: H_{\mathbb{Z}}^{2} \rightarrow H_{Y, \mathbb{Z}}^{3}$ of (2.2). Denoting by $P: F^{1} \mathscr{H}^{2} \rightarrow B$ the composite of the bundle map $F^{1} \mathscr{H}^{2} \rightarrow U$ and the map $p: U \rightarrow B$, the section $r_{\mathbb{Z}}$ allows us to construct a section

$$
s \in \frac{P^{*} \mathscr{H}^{3}}{F^{2} \mathscr{H}^{3}}
$$

over $F^{1} \mathscr{H}_{\mid V}^{2}$ as follows: If $(v, \lambda) \in F^{1} \mathscr{H}^{2}$, that is, $\lambda \in F^{1} H^{2}\left(S_{v}\right)_{0}$, let $\lambda_{F}$ be a lifting of $\lambda$ in $F^{2} H^{3}\left(Y_{v}\right)$. Then we define

$$
s(v, \lambda)=\lambda_{F}-r_{\mathbb{Z}}(\lambda) \bmod . F^{2} H^{3}\left(X_{v}\right) .
$$

This is a well-defined element of $H^{3}\left(X_{v}, \mathbb{C}\right) / F^{2} H^{3}\left(X_{v}\right)$, since clearly $\lambda_{F}-r_{\mathbb{Z}}(\lambda)$ belongs to $H^{3}\left(X_{v}, \mathbb{C}\right)$ and $\lambda_{F}(v)$ is defined up to $F^{2} H^{3}\left(X_{v}\right)$.

In fact, we are mainly interested with the restriction of $s$ to the subvarieties $\phi^{-1}\left(\lambda_{0}\right) \cong V_{\lambda}$. We may then consider these sections of $p^{* \mathscr{H}^{3}} / F^{2} \mathscr{H}_{\mid V_{\lambda}}^{3}$ as the complexified Abel-Jacobi map, as we explain now.

Suppose that $\lambda \in H^{2}\left(S_{v}, \mathbb{Z}\right)_{0} \cap F^{1} H^{2}\left(S_{v}\right)$. Then $\lambda=\left[D_{\lambda}\right]$ for some divisor $D_{\lambda}$ on $S_{v}$, and $j_{v *}\left(D_{\lambda}\right)$ is a one-cycle homologous to zero on $X_{v}$. It is then well known that the element

$$
\Phi_{X_{v}}\left(j_{v *}\left(D_{\lambda}\right)\right) \in J\left(X_{v}\right)=H^{3}\left(X_{v}, \mathbb{C}\right) / F^{2} H^{3}\left(X_{v}\right) \oplus H^{3}\left(X_{v}, \mathbb{Z}\right)
$$


is equal to $\lambda_{F}-r_{\mathbb{Z}}(\lambda) \bmod . F^{2} H^{3}\left(X_{v}\right) \oplus H^{3}\left(X_{v}, \mathbb{Z}\right)$. (The fact that we consider it modulo $H^{3}\left(X_{v}, \mathbb{Z}\right)$ makes it independent of the retraction $r_{\mathbb{Z}}$.) In other words, for integral $\lambda$ we find that $s_{\mid V_{\lambda}} \bmod . H_{\mathbb{Z}}^{3}$ is equal to the section $v_{\lambda}$ of the pullback to $V_{\lambda}$ of the family of intermediate Jacobians $J\left(X_{b}\right)_{b \in B}$ given by

$$
v_{\lambda}(v)=\Phi_{X_{v}}\left(j_{v *}\left(D_{\lambda}\right)\right) \in J\left(X_{v}\right), \quad v \in V_{\lambda} .
$$

We now want to study the infinitesimal properties of the map $\phi$ defined in (2.0) or equivalently of the varieties $V_{\lambda}$. Recall that for $v \in U, \lambda \in H^{1,1}\left(S_{v}\right)_{0}$ we have the map

$$
\bar{\nabla}^{S}(\lambda): H^{1}\left(T_{S_{u}}\right)=T_{U, u} \longrightarrow H^{2}\left(\mathcal{O}_{S_{u}}\right),
$$

which induces

$$
\bar{\nabla}^{S}(\lambda): H^{0}\left(L_{u \mid S_{u}}\right)=\operatorname{Ker} p_{*} \subset T_{U, u} \longrightarrow H^{2}\left(\mathcal{O}_{S_{u}}\right) .
$$

Note that by Serre duality and because $K_{X_{v}}$ is trivial, both spaces have the same dimension. We have the following lemma.

LEMma 4. The following are equivalent:

(i) $\bar{\nabla}^{S}(\lambda): H^{0}\left(L_{u \mid S_{u}}\right) \rightarrow H^{2}\left(\mathbb{O}_{S_{u}}\right)$ is an isomorphism;

(ii) for any $\tilde{\lambda} \in F^{1} H^{2}\left(S_{v}\right)_{0}$ projecting to $\lambda$ modulo $F^{2} H^{2}\left(S_{v}\right)$, the map

$$
(P, \phi): F^{1} \mathscr{H}_{0}^{2} \longrightarrow B \times H^{2}\left(S_{v_{0}}, \mathbb{C}\right)_{0}
$$

is étale at $\tilde{\lambda}$.

Proof. We may clearly assume that $u=v_{0}$ since the change of base point simply composes $\phi$ with the natural isomorphism $H^{2}\left(S_{v_{0}}\right)_{0} \cong H^{2}\left(S_{u}\right)_{0}$. Consider $(P, \phi)_{*}$ : $T_{F^{1} \mathscr{H}^{2}, \tilde{\lambda}} \rightarrow T_{B, p(u)} \times T_{H^{2}\left(S_{u}\right)_{0}, \phi(\tilde{\lambda})}$. Since on $F^{1} H^{2}\left(S_{u}\right)_{0} \subset T_{F^{1} \mathscr{H}^{2}, \tilde{\lambda}}$ this map is simply the inclusion

$$
F^{1} H^{2}\left(S_{u}\right)_{0} \subset H^{2}\left(S_{u}\right)_{0}=T_{H^{2}\left(S_{u}\right)_{0}, \phi(\tilde{\lambda})},
$$

this map induces

$$
(P, \phi)_{*}^{0,2}: T_{U, u} \longrightarrow T_{B, p(u)} \times H^{2}\left(\mathcal{O}_{S_{u}}\right) .
$$

It is then immediate, using the definition of $\bar{\nabla}^{S}$, to show that $(P, \phi)_{*}^{0,2}=\left(p_{*}, \bar{\nabla}^{S}\right)$. But $(P, \phi)_{*}$ is an isomorphism if and only if $(P, \phi)_{*}^{0,2}$ is an isomorphism. Since $p_{*}$ is surjective, this is also equivalent to $(P, \phi)_{*}^{0,2} \mid \operatorname{Ker} p_{*}$ being an isomorphism onto $H^{2}\left(\mathscr{O}_{S_{u}}\right)$, that is, to $\bar{\nabla}^{S}(\lambda): H^{0}\left(L_{u \mid S_{u}}\right) \rightarrow H^{2}\left(\mathcal{O}_{S_{u}}\right)$ being an isomorphism. So Lemma 4 is proved.

In fact, the proof shows the following lemma.

Lemma 5. The kernel of $(P, \phi)_{*}$ at $\tilde{\lambda}$ identifies naturally via the projection to $T_{U, u}$ to

$$
\operatorname{Ker} \bar{\nabla}^{S}(\lambda): H^{0}\left(L_{u \mid S_{u}}\right) \longrightarrow H^{2}\left(\mathcal{O}_{S_{u}}\right)
$$


that is, to the vertical part $T_{V_{\tilde{\bar{\tau}}}^{p(u)}}$ of $T_{V_{\tilde{\bar{\lambda}}}}$, where $V_{\tilde{\lambda}}^{p(u)}$ is the intersection of $V_{\tilde{\lambda}}$ with $\mathbb{P}\left(H^{0}\left(L_{p(u)}\right)\right)=p^{-1}(p(u))$. The reverse isomorphism is given by the differential of the natural section $\tilde{\lambda}$ of $F^{1} \mathscr{H}^{2}$ on $V_{\tilde{\lambda}}^{p(u)}$.

We now study the infinitesimal variation of mixed Hodge structure of the family 甲 $\stackrel{\pi_{Y}}{\rightarrow} U$. It is described as above, by transversality, by a series of maps

$$
\bar{\nabla}^{Y}: F^{i} / F^{i+1} \mathscr{H}_{Y}^{3} \longrightarrow F^{i-1} / F^{i} \mathscr{H}_{Y}^{3} \otimes \Omega_{U},
$$

which fit into the commutative diagram

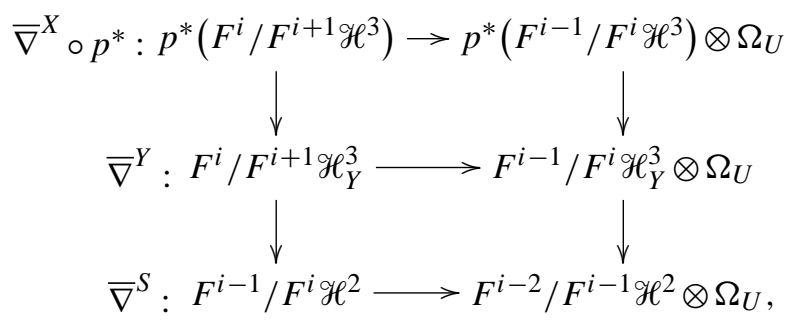

where the first vertical maps are injective and the last ones are surjective. Composing $\bar{\nabla}^{Y}$ with the restriction map $\Omega_{U, u} \rightarrow H^{0}\left(L_{u \mid S_{u}}\right)^{*}$ then gives a map

$$
F^{2} / F^{3} H^{3}\left(Y_{u}\right) \longrightarrow \operatorname{Hom}\left(H^{0}\left(L_{u \mid S_{u}}\right), F^{1} / F^{2} H^{3}\left(Y_{u}\right)\right),
$$

which obviously factors through $F^{1} / F^{2} H^{2}\left(S_{u}\right)_{0}$ since the composition of $p^{*}$ with the restriction to $H^{0}\left(L_{u \mid S_{u}}\right)=\operatorname{Ker} p_{*}$ is zero. (This simply means that there is no variation of Hodge structure for $X$ in the fibers of $p$.) So we have constructed a map

$$
\mu_{0}: H^{1}\left(\Omega_{S_{u}}\right)_{0} \longrightarrow \operatorname{Hom}\left(H^{0}\left(L_{u \mid S_{u}}\right), F^{1} / F^{2} H^{3}\left(Y_{u}\right)\right),
$$

which induces

$$
\mu_{1}: H^{0}\left(L_{u \mid S_{u}}\right) \longrightarrow \operatorname{Hom}\left(H^{1}\left(\Omega_{S_{u}}\right)_{0}, F^{1} / F^{2} H^{3}\left(Y_{u}\right)\right) .
$$

We then have the following.

Lemma 6. There is a natural isomorphism (depending on the choice of a trivialization of $K_{X_{u}}$ )

$$
F^{1} / F^{2} H^{3}\left(Y_{u}\right) \cong\left(T_{U, u}\right)^{*}=\left(H^{1}\left(T_{S_{u}}\right)\right)^{*}
$$

such that for any $\eta \in H^{0}\left(L_{u \mid S_{u}}\right) \cong H^{0}\left(K_{S_{u}}\right)$, the map

$$
{ }^{t}\left(\mu_{1}(\eta)\right): T_{U, u} \longrightarrow H^{1}\left(\Omega_{S_{u}}\right)_{0}
$$

identifies to $\bar{\nabla}^{S}(\eta)$. 
Notice that in the identification $H^{0}\left(L_{u \mid S_{u}}\right) \cong H^{0}\left(K_{S_{u}}\right)$, we use the same trivialization of $K_{X_{u}}$.

Proof. Recall the isomorphisms $T_{U, u}=H^{1}\left(T_{S_{u}}\right)=H^{1}\left(T_{X_{u}}^{S_{u}}\right)$ of Lemma 1. Now $T_{X_{u}}^{S_{u}}$ is dual to $\Omega_{X_{u}}\left(\log S_{u}\right)$, so that, choosing a trivialization of $K_{X_{u}}$, we get an isomorphism $H^{1}\left(T_{X_{u}}^{S_{u}}\right) \cong\left(H^{2}\left(\Omega_{X_{u}}\left(\log S_{u}\right)\right)\right)^{*}$, and taking into account the natural isomorphism (see [5]) $H^{2}\left(\Omega_{X_{u}}\left(\log S_{u}\right)\right)=F^{1} / F^{2} H^{3}\left(Y_{u}\right)$, we get the first assertion.

Next it is known (this is an easy generalization of [9]) that the map

$$
\bar{\nabla}^{Y}: F^{2} / F^{3} \mathscr{H}_{Y}^{3} \longrightarrow F^{1} / F^{2} \mathscr{H}_{Y}^{3} \otimes \Omega_{U}
$$

identifies to the map given by the interior product

$$
H^{1}\left(\Omega_{X_{u}}^{2}\left(\log S_{u}\right)\right) \longrightarrow \operatorname{Hom}\left(H^{1}\left(T_{X_{u}}^{S_{u}}\right), H^{2}\left(\Omega_{X_{u}}\left(\log S_{u}\right)\right)\right) .
$$

The image of the map (2.5) is contained in the set of symmetric homomorphisms from $H^{1}\left(T_{X_{u}}^{S_{u}}\right)$ to its dual; indeed the dual of (2.5) is equal to the (symmetric) cup product

$$
H^{1}\left(T_{X_{u}}^{S_{u}}\right) \otimes H^{1}\left(T_{X_{u}}^{S_{u}}\right) \longrightarrow H^{2}\left(\bigwedge^{2} T_{X_{u}}^{S_{u}}\right)
$$

taking into account the isomorphism $\bigwedge^{2} T_{X_{u}}^{S_{u}}=\left(\Omega_{X_{u}}^{2}\left(\log S_{u}\right)\right)^{*}$, the triviality of $K_{X_{u}}$, and Serre duality.

It follows that for $\lambda \in H^{1}\left(\Omega_{X_{u}}^{2}\left(\log S_{u}\right)\right), \eta, \chi \in H^{1}\left(T_{X_{u}}^{S_{u}}\right)$, we have

$$
\left\langle\bar{\nabla}^{Y}(\lambda)(\eta), \chi\right\rangle=\left\langle\bar{\nabla}^{Y}(\lambda)(\chi), \eta\right\rangle
$$

Now note that the inclusion $H^{0}\left(L_{u \mid S_{u}}\right) \hookrightarrow H^{1}\left(T_{X_{u}}^{S_{u}}\right)$ is dual to the residue map Res: $H^{2}\left(\Omega_{X_{u}}\left(\log S_{u}\right)\right) \rightarrow H^{2}\left({ }_{S_{S}}\right)$, so that for $\eta \in H^{0}\left(L_{u \mid S_{u}}\right)$, (2.6) gives

$$
\left\langle\bar{\nabla}^{Y}(\lambda)(\eta), \chi\right\rangle=\left\langle\operatorname{Res}\left(\bar{\nabla}^{Y}(\lambda)(\chi)\right), \eta\right\rangle,
$$

where the second pairing in (2.7) is the duality between $H^{0}\left(L_{u \mid S_{u}}\right)$ and $H^{2}\left(\mathscr{O}_{S_{u}}\right)$. (We always use the same trivialization of $K_{X_{u}}$ to compute the pairings.) But by diagram (2.4), we have $\left.\operatorname{Res}\left(\bar{\nabla}^{Y}(\lambda)(\chi)\right)=\bar{\nabla}^{S}(\operatorname{Res}(\lambda))(\chi)\right)$ and by definition of $\mu_{1}$, we have $\bar{\nabla}^{Y}(\lambda)(\eta)=\mu_{1}(\eta)(\operatorname{Res}(\lambda))$. So we have proved for any $\lambda \in H^{1}\left(\Omega_{S_{u}}\right)_{0}$, for any $\eta \in H^{0}\left(L_{u \mid S_{u}}\right)$ and $\chi \in H^{1}\left(T_{X_{u}}^{S_{u}}\right)$, the equality

$$
\left\langle\mu_{1}(\eta)(\lambda), \chi\right\rangle=\left\langle\bar{\nabla}^{S}(\lambda)(\chi), \eta\right\rangle,
$$

where the first pairing is the duality above between $H^{1}\left(T_{X_{u}}^{S_{u}}\right)$ and $H^{2}\left(\Omega_{X_{u}}\left(\log S_{u}\right)\right)$, while the second one is the duality between $H^{0}\left(L_{u \mid S_{u}}\right)$ and $H^{2}\left({ }_{S_{u}}\right)$. Finally, for 
$\eta \in H^{0}\left(L_{u \mid S_{u}}\right) \cong H^{0}\left(K_{S_{u}}\right), \lambda \in H^{1}\left(\Omega_{S_{u}}\right)_{0}, \chi \in H^{1}\left(T_{X_{u}}^{S_{u}}\right)$, we have the equalities

$$
\left\langle{ }^{t}\left(\bar{\nabla}^{S}(\eta)\right)(\lambda), \chi\right\rangle=\left\langle\lambda, \bar{\nabla}^{S}(\eta)(\chi)\right\rangle=\left\langle\bar{\nabla}^{S}(\lambda)(\chi), \eta\right\rangle \stackrel{(2.8)}{=}\left\langle\mu_{1}(\eta)(\lambda), \chi\right\rangle,
$$

where the second equality is standard and follows from the fact that the intersection pairing on $H^{2}\left(S_{u}\right)_{0}$ is flat with respect to the Gauss-Manin connection and that, for this pairing, $H^{2,0}\left(S_{u}\right)$ is perpendicular to $F^{1} H^{2}\left(S_{u}\right)_{0}$. This proves that ${ }^{t}\left(\bar{\nabla}^{S}(\eta)\right)=\mu_{1}(\eta)$, as we wanted.

We conclude this section with the "complexified infinitesimal Abel-Jacobi map." Recall that from Lemma 5 we get, for $\lambda \in H^{1}\left(\Omega_{S_{u}}\right)_{0}$ with lifting $\tilde{\lambda} \in F^{1} H^{2}\left(S_{u}\right)_{0}$, a natural identification between $\operatorname{Ker} \bar{\nabla}^{S}(\lambda)_{\mid H^{0}\left(L_{u \mid S_{u}}\right)}$ and $\operatorname{Ker} P_{*}: T_{V_{\tilde{\lambda}} \times \tilde{\lambda}, \tilde{\lambda}} \rightarrow T_{B, p(u)}$, where by definition of $V_{\tilde{\lambda}}, V_{\tilde{\lambda}} \times \tilde{\lambda} \subset U \times H^{2}\left(S_{u_{0}}, \mathbb{C}\right)_{0}$ is in fact contained in $F^{1} \mathscr{H}^{2}$. Now consider the section $s$ of the bundle $P^{*} \mathscr{H}^{3} / F^{2} \mathscr{H}^{3}$ constructed in (2.3). Since this bundle is naturally trivial on the fibers of $P$, it makes sense to differentiate $s_{\mid V_{\tilde{\lambda}} \times \tilde{\lambda}}$ in the direction contained in $\operatorname{Ker} P_{*}$. It follows that we have a map

$$
d s: \operatorname{Ker} P_{*}=\operatorname{Ker} \bar{\nabla}^{S}(\lambda)_{\mid H^{0}\left(L_{u \mid S_{u}}\right)} \longrightarrow H^{3}\left(X_{u}\right) / F^{2} H^{3}\left(X_{u}\right) .
$$

On the other hand, the map

$$
\mu_{0}: H^{1}\left(\Omega_{S_{u}}\right)_{0} \longrightarrow \operatorname{Hom}\left(H^{0}\left(L_{u \mid S_{u}}\right), F^{1} / F^{2} H^{3}\left(Y_{u}\right)\right)
$$

satisfies Res $\circ \mu_{0}(\lambda)=\bar{\nabla}^{S}(\lambda)_{\mid H^{0}\left(L_{u \mid S_{u}}\right)}$ and hence induces a map

$$
\mu_{2}: \operatorname{Ker} \bar{\nabla}^{S}(\lambda)_{\mid H^{0}\left(L_{u \mid S_{u}}\right)} \longrightarrow H^{3}\left(X_{u}\right) / F^{2} H^{3}\left(X_{u}\right) .
$$

Now we have the following.

LEMma 7. We have the equality

$$
d s=\mu_{2} .
$$

Proof. Indeed, recall that $s_{\mid V_{\tilde{\lambda}} \times \tilde{\lambda}}$ is equal to $\tilde{\lambda}_{F}-r_{\mathbb{Z}}(\tilde{\lambda}) \bmod . F^{2} \mathscr{H}^{3}$, where $\tilde{\lambda}_{F}$ is any lifting of $\tilde{\lambda} \in F^{1} \mathscr{H}_{\mid V_{\tilde{\lambda}}}^{2}$ in $F^{2} \mathscr{H}_{Y \mid V_{\tilde{\lambda}}}^{3}$. Since $\tilde{\lambda}$ is flat, we have

$$
\nabla^{X}\left(\tilde{\lambda}_{F}-r_{\mathbb{Z}}(\tilde{\lambda})\right)=\nabla^{Y}\left(\tilde{\lambda}_{F}\right)
$$

since $\tilde{\lambda}_{F}$ is a section of $F^{2} \mathscr{H}_{Y \mid V_{\tilde{\lambda}}}^{3}$, we have, by definition of $\bar{\nabla}^{Y}$,

$$
\nabla^{Y}\left(\tilde{\lambda}_{F}\right) \bmod . F^{2} \mathscr{H}_{Y}^{3}=\bar{\nabla}^{Y}\left(\bar{\lambda}_{F}\right),
$$


where $\bar{\lambda}_{F}$ is the projection of $\tilde{\lambda}_{F}$ in $F^{2} \mathscr{H}_{Y}^{3} / F^{3} \mathscr{H}_{Y}^{3}$. But then for $h \in T_{\tilde{\tilde{\lambda}}_{\tilde{\lambda}}, u} \cap \operatorname{Ker} p_{*}=$ $\operatorname{Ker} \bar{\nabla}^{S}(\lambda)_{\mid H^{0}\left(L_{u \mid S_{u}}\right)}$, we have

$$
d s(h)=\nabla_{h}^{X}\left(\tilde{\lambda}_{F}-r_{\mathbb{Z}}(\tilde{\lambda})\right) \bmod . F^{2} H^{3}\left(X_{u}\right)=\bar{\nabla}^{Y}\left(\bar{\lambda}_{F}\right)(h),
$$

and by definition of $\mu_{2}$ the right-hand side is equal to $\mu_{2}(h)$.

The reason we call $d s$ or $\mu_{2}$ the complexified infinitesimal Abel-Jacobi map is, again, that if $\tilde{\lambda}$ is an integral class, we have shown that $s_{\mid V_{\tilde{\lambda}} \times \tilde{\lambda}}$ is a lifting of the normal function

$$
v_{\tilde{\lambda}}(u)=\Phi_{X_{u}}\left(j_{S_{u *}}\left(D_{\tilde{\lambda}, u}\right)\right) \in J\left(X_{u}\right)
$$

to a section of $p^{*}\left(\mathscr{H}^{3} / F^{2} \mathscr{H}^{3}\right)$ on $V_{\tilde{\lambda}}$. Then if $h \in \operatorname{Ker} p_{*}, d s(h)$ is simply the differential of $v_{\tilde{\lambda}}$ in the direction $h$, which makes sense since $X_{u}$, and hence $J\left(X_{u}\right)$ remains constant in the direction $h$.

3. An infinitesimal criterion for the nonfinite generation of the image of the Abel-Jacobi map. With the notation of Section 2, we now assume that $\operatorname{dim} B>0$. Recall that we have defined for $S_{u} \subset X_{u}$ and for $\lambda \in H^{1}\left(\Omega_{S_{u}}\right)_{0}, \eta \in H^{0}\left(K_{S_{u}}\right)$ the maps

$$
\begin{aligned}
& \bar{\nabla}^{S}(\eta): H^{1}\left(T_{S_{u}}\right) \longrightarrow H^{1}\left(\Omega_{S_{u}}\right)_{0}, \\
& \bar{\nabla}^{S}(\lambda): H^{1}\left(T_{S_{u}}\right) \longrightarrow H^{2}\left(\mathcal{O}_{S_{u}}\right) .
\end{aligned}
$$

We prove in this section the following infinitesimal criterion for the infinite generation of the Griffiths group of the general fiber $X_{b}$.

Proposition 1. Assume that $L_{u}$ is sufficiently ample and that for generic $u \in U$ and generic $\eta \in H^{0}\left(K_{S_{u}}\right)$, we have that

(i) the map $\bar{\nabla}^{S}(\eta): H^{1}\left(T_{S_{u}}\right) \rightarrow H^{1}\left(\Omega_{S_{u}}\right)_{0}$ is injective;

(ii) for generic $\lambda \in H^{1}\left(\Omega_{S_{u}}\right)_{0}$ such that $\bar{\nabla}^{S}(\lambda)(\eta)=0$, we have that

$$
\operatorname{Ker} \bar{\nabla}^{S}(\lambda): H^{0}\left(L_{u \mid S_{u}}\right) \longrightarrow H^{2}\left({ }^{O_{S_{u}}}\right)
$$

is generated by $\eta$.

Then for the general point $t \in B$, the Abel-Jacobi map $\phi_{X_{t}}$ of $X_{t}$ satisfies that $\operatorname{Im} \Phi_{X_{t}} \otimes \mathbb{Q}$ is an infinite-dimensional $\mathbb{Q}$-vector space.

In assumption (ii), $\eta$ is viewed as an element of $H^{0}\left(L_{u \mid S_{u}}\right) \subset H^{1}\left(T_{S_{u}}\right)$.

To start the proof, we first note the following lemma.

Lemma 8. Assumption (ii) implies that for generic $u$ and generic $\lambda \in H^{1}\left(\Omega_{S_{u}}\right)_{0}$, the map

$$
\bar{\nabla}^{S}(\lambda): H^{0}\left(L_{u \mid S_{u}}\right) \longrightarrow H^{2}\left(\mathcal{O}_{S_{u}}\right)
$$

is an isomorphism. 
Proof. Identifying $H^{0}\left(L_{u \mid S_{u}}\right)$ with $H^{0}\left(K_{S_{u}}\right)$ by a trivialization of $K_{X_{u}}$, the maps $\bar{\nabla}^{S}(\lambda): H^{0}\left(L_{u \mid S_{u}}\right) \rightarrow H^{2}\left(\mathcal{O}_{S_{u}}\right)$ are symmetric, with respect to Serre duality. Hence $\bar{\nabla}^{S}(\lambda)$ determines a quadric $q_{\lambda}$ on $\mathbb{P}\left(H^{0}\left(L_{u \mid S_{u}}\right)\right)$. If $\lambda$ is as in assumption (ii), the quadric $q_{\lambda}$ has $\eta$ for only singular point, and since $\eta$ is generic, it is not in the base locus of the system of quadrics $q_{\lambda}$. Hence the tangent space at $\lambda$ to the discriminant hypersurface, parametrizing singular quadrics $q_{\lambda}$ being equal to the set of $q_{\lambda}$ vanishing at $\eta$, is a proper subspace of $H^{1}\left(\Omega_{S_{u}}\right)_{0}$, so the generic $q_{\lambda}$ is smooth.

Now note that the condition that $\bar{\nabla}^{S}(\lambda): H^{0}\left(L_{u \mid S_{u}}\right) \rightarrow H^{2}\left(\mathscr{O}_{S_{u}}\right)$ is an isomorphism is Zariski open on $H^{1}\left(\Omega_{S_{u}}\right)_{0}$, which is the complexification of $H_{\mathbb{R}}^{1,1}\left(S_{u}\right)_{0}:=$ $H^{1,1}\left(S_{u}\right) \cap H^{2}\left(S_{u}, \mathbb{R}\right)_{0}$. So if it is satisfied at some point, it will be satisfied at some real point $\lambda \in H_{\mathbb{R}}^{1,1}\left(S_{u}\right)_{0}$, which obviously has a natural (real) lifting $\lambda$ in $F^{1} H^{2}\left(S_{u}\right)_{0}$.

From Lemma 4 we know that at such a $\lambda \in F^{1} \mathscr{H}^{2}$ the map

$$
(P, \phi): F^{1} \mathscr{H}^{2} \longrightarrow B \times H^{2}\left(S_{u_{0}}, \mathbb{C}\right)_{0}
$$

is étale, so it is a local isomorphism for the usual topology. Hence there are open connected neighbourhoods $B^{\prime} \subset B$ of $p(u), V^{\prime} \subset H^{2}\left(S_{u_{0}}, \mathbb{C}\right)_{0}$ of $\phi(\lambda)$, and $W \subset$ $F^{1} \mathscr{H}^{2}$ of $\lambda$, with $W \stackrel{(P, \phi)}{\cong} B^{\prime} \times V^{\prime}$. Finally, note that since $\phi(\lambda)$ is real, the rational points in $V^{\prime} \cap H^{2}\left(S_{u_{0}}, \mathbb{Q}\right)_{0}$ are Zariski dense in $V^{\prime}$. For any such rational point $\lambda \in V^{\prime}$, the fiber $\phi^{-1}(\lambda) \cap W$ is then naturally isomorphic to $B^{\prime}$ by $P$, and it parametrizes then the pairs $S_{t} \stackrel{j_{t}}{\hookrightarrow} X_{t}$ such that $\lambda_{t}$ is algebraic on $S_{t}$. For each such $\lambda$, we choose an integer $m_{\lambda}$ such that $m_{\lambda} \lambda$ is integral, and then $m_{\lambda} \lambda=c_{1}\left(D_{\lambda, t}\right)$ on $S_{t}$. Hence we get a normal function $v_{\lambda}$ on $B^{\prime}$, that is, a section of the sheaf

$$
\mathscr{g}=\mathscr{H}^{3} / F^{2} \mathscr{H}^{3} \oplus H_{\mathbb{Z}}^{3}
$$

defined by

$$
v_{\lambda}(t)=\Phi_{X_{t}}\left(j_{t *}\left(D_{\lambda, t}\right)\right) \in J\left(X_{t}\right) .
$$

We use the countably many $v_{\lambda}, \lambda \in V_{\mathbb{Q}}^{\prime}$, in order to prove Proposition 1 . So we assume by contradiction the following assumption:

(*) For any general point $t \in B^{\prime}$, the image of $\Phi_{X_{t}}$ tensorized by $\mathbb{Q}$ is finitely generated.

Then we have the following.

Lemma 9. If $(*)$ holds, there exists $\lambda_{1}, \ldots, \lambda_{N} \in V_{\mathbb{Q}}^{\prime}$ such that for any $\lambda \in V_{\mathbb{Q}}^{\prime}$, there exist integers $m \neq 0, m_{1}, \ldots, m_{N}$, satisfying the equality

$$
m v_{\lambda}=\sum_{i} m_{i} v_{\lambda_{i}} \text { in } \mathscr{g} .
$$

Proof. Choose an ordering $\lambda_{i}, i \in \mathbb{N}$, of the elements of $V_{\mathbb{Q}}^{\prime}$. For any sequence $\left(\alpha_{i}\right)_{i \in \mathbb{N}}$ of integers with only finitely many nonzero elements, let 


$$
B_{\alpha .}^{\prime}=\left\{t \in B^{\prime}, \sum_{i} \alpha_{i} v_{\lambda_{i}}(t)=0 \text { in } J\left(X_{t}\right)\right\} .
$$

Then $B_{\alpha .}^{\prime}$ is an analytic subset of $B^{\prime}$, so any point in

$$
B^{\prime \prime}=B^{\prime}-\bigcup_{B_{\alpha .}^{\prime} \neq B^{\prime}} B_{\alpha}^{\prime}
$$

is general. On the other hand, by definition, if $t \in B^{\prime \prime}$, any relation with integral coefficients $\sum_{i} \alpha_{i} v_{\lambda_{i}}(t)=0$ in $J\left(X_{t}\right)$ implies that $\sum_{i} \alpha_{i} v_{\lambda_{i}}=0$ in $g$. Lemma 9 follows, taking any $t \in B^{\prime \prime}$ at which (*) holds.

Coming back to the section $s$ of $\left.P^{*}\left(\mathscr{H}^{3} / F^{2} \mathscr{H ^ { 3 }}\right)_{\mid W}\right)$ defined in (2.3), we get the following corollary.

Corollary 1. Under the same assumption $(*)$, for any $\lambda \in V^{\prime}, s_{\mid B^{\prime} \times \lambda}$ belongs to the finite vector space $K$ of holomorphic sections of $\left(\mathscr{H}^{3} / F^{2} \mathscr{H}^{3}\right)_{\mid B^{\prime}}$ generated by the image of $H_{\mathbb{C}_{\mid B^{\prime}}}^{3}$ in $\left(\mathscr{H}^{3} / F^{2} \mathscr{H}^{3}\right)_{\mid B^{\prime}}$ and by liftings $\tilde{v}_{\lambda_{i}}$ of $v_{\lambda_{i}}$ in $\left(\mathscr{H}^{3} / F^{2} \mathscr{H}^{3}\right)_{\mid B^{\prime}}$ for $i=1, \ldots, N$.

Here we use the isomorphism $W \cong B^{\prime} \times V^{\prime}$ given by $(P, \phi)$.

Proof. By Lemma 9, the conclusion is true for $\lambda \in V_{\mathbb{Q}}^{\prime}$. Indeed, a relation $m v_{\lambda}=$ $\sum_{i} m_{i} v_{\lambda_{i}}$ in $\mathscr{F}$ is equivalent to a relation

$$
m \tilde{v}_{\lambda}=\sum_{i} m_{i} \tilde{v}_{\lambda_{i}}+\alpha \quad \text { in }\left(\frac{\mathscr{H}^{3}}{F^{2} \mathscr{H}^{3}}\right)_{\mid B^{\prime}},
$$

where $\alpha \in H_{\mathbb{Z}}^{3}$ and $\tilde{v}_{\lambda_{i}}, \tilde{v}_{\lambda}$ are liftings of our normal functions in $\left(\mathscr{H}^{3} / F^{2} \mathscr{H}^{3}\right)_{\mid B^{\prime}}$. On the other hand, we have shown in Section 2 that $m_{\lambda} S_{\mid B^{\prime} \times \lambda}, m_{\lambda_{i}} s_{\mid B^{\prime} \times \lambda_{i}}$ give such liftings.

In order to deduce from this that the conclusion is true for any $\lambda \in V^{\prime}$, we use now the Zariski density of $V_{\mathbb{Q}}^{\prime}$ in $V^{\prime}$. To be precise, using a trivialization of the bundle $\left(\mathscr{H}^{3} / F^{2} \mathscr{H}^{3}\right)_{\mid B^{\prime}}$, Corollary 1 will follow now from the next lemma.

Lemma 10. Let $K$ be a finite-dimensional set of functions on $B^{\prime}$. Let $f$ be a function on $B^{\prime} \times V^{\prime}$, where $V^{\prime}$ is a connected open set of $\mathbb{C}^{k}$ meeting $\mathbb{R}^{k}$, such that for any $\lambda \in V^{\prime} \cap \mathbb{Q}^{k}, f_{\mid B^{\prime} \times \lambda} \in K$. Then for any $\lambda \in V^{\prime}, f_{\mid B^{\prime} \times \lambda} \in K$.

Proof. Let $k=\operatorname{dim} K$ and let $p_{1}, \ldots, p_{k}$ be points on $B^{\prime}$ such that the restriction map $K \rightarrow \oplus_{i} \hat{O}_{p_{i}}$ is an isomorphism. Then we have a basis $\left(k_{i}\right)$ of $K$ such that $k_{i}\left(p_{j}\right)=\delta_{i j}$. So any element $g$ of $K$ satisfies $g=\sum_{i} g\left(p_{i}\right) k_{i}$. It follows that the function $f(b, v)-\sum_{i} f\left(p_{i}, v\right) k_{i}(b)$ on $B^{\prime} \times V^{\prime}$ vanishes on $B^{\prime} \times\left(V^{\prime} \cap \mathbb{Q}^{k}\right)$, hence everywhere, by the (analytic) Zariski density of $B^{\prime} \times\left(V^{\prime} \cap \mathbb{Q}^{k}\right)$ in $B^{\prime} \times V^{\prime}$.

Now we use analytic continuation to conclude the following. 
COROllary 2. Let $U^{\prime}$ be any open subset of $U$ contained in the image of the projection $W \rightarrow U$. (We recall that $W$ is an open subset of $F^{1} \mathscr{H}_{\mid V}^{2}$ and that $V$ is open in $U$.) Then under the same assumption (*), for any $\lambda_{u} \in F^{1} \mathscr{H}_{\mid U^{\prime}}^{2}$ such that $(P, \phi)$ is étale at $\lambda_{u}$, the section $s_{\mid U_{\lambda_{u}, 0}^{\prime}}$ belongs to $P^{*}(K)$, where $U_{\lambda_{u}, 0}^{\prime}$ denotes the irreducible component of $U_{\lambda_{u}}^{\prime} \cong \phi^{-1}\left(\phi\left(\lambda_{u}\right)\right)$ containing $u$ (which is unic since by hypothesis $U_{\lambda_{u}}^{\prime}$ is smooth at $u$ ).

Proof. Let $\left(F^{1} \mathscr{H}_{\mid U^{\prime}}^{2}\right)_{\text {et }}$ denote the Zariski-dense open subset of $F^{1} \mathscr{H}_{\mid U^{\prime}}^{2}$ where $(P, \phi)$ is étale. We can cover $\left(F^{1} \mathscr{H}_{\mid U^{\prime}}^{2}\right)_{\mathrm{et}}$ by connected open sets $W_{i}$ isomorphic to $B_{i} \times V_{i}$ by $(P, \phi)$ for some open subsets $B_{i}$ of $B^{\prime}$ and $V_{i}$ of $H^{2}\left(\left(S_{u_{0}}, \mathbb{C}\right)_{0}\right)$. Then for $\lambda_{u} \in W_{i}, B_{i} \times \phi\left(\lambda_{u}\right)$ is open in $U_{\lambda_{u}, 0}^{\prime}$. So if we show that $s_{\mid B_{i} \times \phi\left(\lambda_{u}\right)}$ belongs to $K$, there is a $k \in K$ such that

$$
P^{*} k_{\mid B_{i} \times \phi\left(\lambda_{u}\right)}=s_{\mid B_{i} \times \phi\left(\lambda_{u}\right)},
$$

and this will be true everywhere on $U_{\lambda_{u}, 0}^{\prime}$ by analytic continuation.

So it suffices to prove the following: For any $W_{i} \cong B_{i} \times V_{i}$, we have that for any $\lambda \in V_{i}, s_{\mid B_{i} \times \lambda}$ belongs to $K$. But using the same argument as in Corollary 1 , we see that if this is true for $W_{i}$ and if $W_{i} \cap W_{j} \neq \emptyset$, this is true as well for $W_{j}$. Since this is true on $W$ by Corollary 1 and $\left(F^{1} \mathscr{H}_{\mid U^{\prime}}^{2}\right)_{\text {et }}$ is connected, this is true for all $W_{i}$. Corollary 2 is proved.

We conclude with the following corollary.

Corollary 3. Let $\lambda_{u} \in F^{1} \mathscr{H}_{\mid U^{\prime}}^{2}$ be such that $U_{\lambda_{u}}^{\prime}$ is irreducible reduced, and generically finite over $B$ via $p$. Then if $(*)$ is satisfied, for any $h \in T_{U_{\lambda_{u}}^{\prime}, \lambda_{u}}$, such that $P_{*}(h)=0$ in $T_{B, p(u)}$, we have $d s(h)=0$ in $H^{3}\left(X_{u}\right) / F^{2} H^{3}\left(X_{u}\right)$.

This is immediate since $P: U_{\lambda_{u}}^{\prime} \rightarrow B$ is a generic isomorphism, that is, $(P, \phi)$ is étale at the generic point of $U_{\lambda_{u}}^{\prime}$. We then can apply the previous corollary and conclude that for some $k \in K$, we have $P^{*}(k)=s$ on some open set of $U_{\lambda_{u}}^{\prime}$. Hence the equality is true everywhere by irreducibility, and it follows that the vertical derivatives of $s_{\mid U_{\lambda}^{\prime}}$ vanish.

Proof of Proposition 1. We now show that the hypotheses of Proposition 1 contradict the conclusion of Corollary 3 . The hypotheses are as follows:

(i) the map $\bar{\nabla}^{S}(\eta): H^{1}\left(T_{S_{u}}\right) \rightarrow H^{1}\left(\Omega_{S_{u}}\right)_{0}$ is injective for generic $u$ and $\eta \in$ $H^{0}\left(K_{S_{u}}\right)$;

(ii) for generic $\lambda \in H^{1}\left(\Omega_{S_{u}}\right)_{0}$, such that $\bar{\nabla}^{S}(\lambda)(\eta)=0$, we have that

$$
\operatorname{Ker} \bar{\nabla}^{S}(\lambda): H^{0}\left(L_{u \mid S_{u}}\right) \longrightarrow H^{2}\left(\mathcal{O}_{S_{u}}\right)
$$

is generated by $\eta$. 
Recall from Lemma 6 that the transposed map

$$
{ }^{t}\left(\bar{\nabla}^{S}(\eta)\right): H^{1}\left(\Omega_{S_{u}}\right)_{0} \longrightarrow\left(H^{1}\left(T_{S_{u}}\right)\right)^{*} \cong \frac{F^{1} H^{3}\left(Y_{u}\right)}{F^{2} H^{3}\left(Y_{u}\right)}
$$

satisfies

$$
\operatorname{Res} \circ^{t}\left(\bar{\nabla}^{S}(\eta)\right)=\bar{\nabla}_{\eta}^{S}: H^{1}\left(\Omega_{S_{u}}\right)_{0} \longrightarrow H^{2}\left(\mathcal{O}_{S_{u}}\right) .
$$

Now hypothesis (i) says that ${ }^{t}\left(\bar{\nabla}^{S}(\eta)\right)$ is surjective; furthermore, the condition $\operatorname{dim} B>$ 0 implies $\operatorname{dim} F^{1} H^{3}\left(X_{u}\right) / F^{2} H^{3}\left(X_{u}\right)>0$. It follows that for generic $\lambda \in \operatorname{Ker} \bar{\nabla}_{\eta}^{S}$, we have ${ }^{t}\left(\bar{\nabla}^{S}(\eta)\right)(\lambda) \neq 0$ in

$$
\frac{F^{1} H^{3}\left(X_{u}\right)}{F^{2} H^{3}\left(X_{u}\right)}=\operatorname{Ker}\left(\operatorname{Res}: \frac{F^{1} H^{3}\left(Y_{u}\right)}{F^{2} H^{3}\left(Y_{u}\right)} \longrightarrow H^{2}\left(\mathcal{O}_{S_{u}}\right)\right) .
$$

Note also that by definition $\bar{\nabla}_{\eta}^{S}(\lambda)=\bar{\nabla}^{S}(\lambda)(\eta) \in H^{2}\left(\mathcal{O}_{S_{u}}\right)$, so we conclude from assumption (ii) that we can find $\lambda$ such that

(a) $\operatorname{Ker} \bar{\nabla}^{S}(\lambda)$ is generated by $\eta$, with $\eta$ generic in $H^{0}\left(L_{u \mid S_{u}}\right)$;

(b) ${ }^{t}\left(\bar{\nabla}^{S}(\eta)\right)(\lambda) \neq 0$ in $F^{1} H^{3}\left(X_{u}\right) / F^{2} H^{3}\left(X_{u}\right)$.

Now recall Lemmas 6 and 7, which say that for $\eta \in \operatorname{Ker} \bar{\nabla}^{S}(\lambda)$, so that $\eta$ is tangent to $V_{\tilde{\lambda}}$ at $u$ for any $\tilde{\lambda} \in F^{1} H^{2}\left(S_{u}\right)_{0}$ over $\lambda$, and $\eta$ is annihilated by $p_{*}$,

$$
{ }^{t}\left(\bar{\nabla}^{S}(\eta)\right)(\lambda) \in \frac{F^{1} H^{3}\left(X_{u}\right)}{F^{2} H^{3}\left(X_{u}\right)}
$$

is equal to $d s_{\mid V_{\tilde{\lambda}}}(\eta)$.

So the hypotheses imply that for any $\tilde{\lambda} \in F^{1} H^{2}\left(S_{u}\right)_{0}$ over $\lambda$, the vertical derivative of $s_{\mid V_{\tilde{\lambda}}}$ is nonzero. In order to contradict Corollary 3, it suffices now to show that we can choose $\tilde{\lambda}$ so that $V_{\tilde{\lambda}}$ is smooth at $u$ and generically finite over $B$.

The first statement follows easily from (a) and (b): indeed, to prove the smoothness of $V_{\tilde{\lambda}}$ at $u$, it suffices to show that

$$
\bar{\nabla}^{S}(\lambda): H^{1}\left(T_{S_{u}}\right) \longrightarrow H^{2}\left(\mathcal{O}_{S_{u}}\right)
$$

is surjective or that its dual

$$
{ }^{t}\left(\bar{\nabla}^{S}(\lambda)\right): H^{0}\left(L_{u \mid S_{u}}\right) \longrightarrow H^{1}\left(T_{S_{u}}\right)^{*}=\frac{F^{1} H^{3}\left(Y_{u}\right)}{F^{2} H^{3}\left(Y_{u}\right)}
$$

is injective.

But one sees easily, as in Section 2, that $\operatorname{Res} \circ^{t}\left(\bar{\nabla}^{S}(\lambda)\right)$ is equal to $\bar{\nabla}^{S}(\lambda)_{\mid H^{0}\left(L_{u \mid S_{u}}\right)}$, and hence its kernel is generated by $\eta$ by (a). Furthermore, one has the equality

$$
{ }^{t}\left(\bar{\nabla}^{S}(\lambda)\right)(\eta)={ }^{t}\left(\bar{\nabla}^{S}(\eta)\right)(\lambda) \in \frac{F^{1} H^{3}\left(X_{u}\right)}{F^{2} H^{3}\left(X_{u}\right)},
$$


and by (b) this is nonzero. So ${ }^{t}\left(\bar{\nabla}^{S}(\lambda)\right)$ is injective, as we wanted to prove.

What remains is to show that for general $\tilde{\lambda}$ lifting $\lambda$, the variety $V_{\tilde{\lambda}}$ is generically finite over $B$ via $P$. Recall from (2.1) that on $V_{\tilde{\lambda}}$ we have a natural section $\tilde{\lambda}^{1,1}$ of the bundle $\mathscr{H}^{1,1}$. Now on the total space of $\mathscr{H}^{1,1}$, let $\mathscr{D}$ be the discriminant hypersurface; that is, for any $u$,

$$
\mathscr{D}_{u}=\left\{\lambda \in \mathscr{H}_{u}^{1,1}, \bar{\nabla}^{S}(\lambda): H^{0}\left(L_{u \mid S_{u}}\right) \longrightarrow H^{2}\left(\mathcal{O}_{S_{u}}\right) \text { is not an isomorphism }\right\} .
$$

If $q: F^{1} \mathscr{H}^{2} \rightarrow \mathscr{H}^{1,1}$ is the natural projection, it follows from Lemma 4 that $q^{-1}(\mathscr{D})$ is equal to the ramification locus of the map $(P, \phi)$. This implies that the ramification locus of the map $P_{\mid V_{\tilde{\lambda}}}$ is equal to $\left(\tilde{\lambda}^{1,1}\right)^{-1}(\mathscr{D})$. Hence $P_{\mid V_{\tilde{\lambda}}}$ is generically finite if and only if $\tilde{\lambda}^{1,1}\left(V_{\tilde{\lambda}}\right)$ is not contained in $\mathscr{D}$. Now since $\eta$ is contained in the vertical tangent space of $V_{\tilde{\lambda}}$ at $u$, it suffices to prove that $\tilde{\lambda}_{*}^{1,1}(\eta)$ is not tangent to $\mathscr{D}_{u}$ at $\lambda$. But the symmetric maps

$$
\bar{\nabla}^{S}(\mu): H^{0}\left(L_{u \mid S_{u}}\right) \longrightarrow H^{2}\left(\mathcal{O}_{S_{u}}\right)
$$

can be viewed as quadrics $q_{\mu}$ on $\mathbb{P}\left(H^{0}\left(L_{u \mid S_{u}}\right)\right)$. Then the assumption on $\lambda$ means that $q_{\lambda}$ has $\eta$ as its only singular point. It follows that the tangent space to $\mathscr{D}_{u}$ at $\lambda$ is the set $\left\{\mu \in H^{1}\left(\Omega_{S_{u}}\right)_{0}, q_{\mu}(\eta)=0\right\}$.

Now we use Lemma 3 and conclude that if $\tilde{\lambda}_{*}^{1,1}(\eta)$ was tangent to $\mathscr{D}_{u}$ at $\lambda$ for any $\tilde{\lambda}$ lifting $\lambda$, the subspace $\bar{\nabla}_{\eta}^{S}\left(H^{0}\left(K_{S_{u}}\right)\right)$ of $H^{1}\left(\Omega_{S_{u}}\right)_{0}$ would be tangent to $\mathscr{D}_{u}$ at $\lambda$. Hence we would have the following: For any $\omega \in H^{0}\left(K_{S_{u}}\right)$,

$$
\left\langle\eta, \bar{\nabla}^{S}\left(\bar{\nabla}_{\eta}^{S}(\omega)\right)(\eta)\right\rangle=0 .
$$

This cannot hold for generic $\eta$ and sufficiently ample $L$ for the following reason: One can show (and this is done in the next section) by describing the variation of Hodge structure of the family of surfaces $S_{u}$ (with fixed $X_{u}$ ) in terms of the Jacobian ring associated to $S_{u} \subset X_{u}$ (see [7] and [10]) that there is a natural surjective map

$$
\psi: H^{0}\left(3 L_{u \mid S_{u}}\right) \longrightarrow H^{2}\left(\mathcal{O}_{S_{u}}\right)
$$

and that (3.9) would mean exactly that $\psi\left(\eta^{3}\right)=0$. But if $L$ is sufficiently ample, the multiplication map $S^{3} H^{0}\left(L_{u}\right) \rightarrow H^{0}\left(3 L_{u}\right)$ is surjective, so that $\psi\left(\eta^{3}\right)=0$ for any $\eta$ would imply that $\psi=0$, which is absurd since $H^{2}\left(\mathcal{O}_{S_{u}}\right) \neq 0$. So we have obtained the desired contradiction with the conclusion of Corollary 3 , and this shows that the finiteness assumption $(*)$ is absurd. The proof of Proposition 1 is now complete.

4. Checking the infinitesimal criterion for any Calabi-Yau threefold. In this section we prove that conditions (i) and (ii) of Proposition 1 are satisfied for a sufficiently large multiple of an ample line bundle on $X$. This will conclude the proof of Theorem 4. We start with the proof of (i). 
Proposition 2. Let X be a Calabi-Yau threefold and $L_{1}$ be a line bundle on X. If $L_{1}$ is sufficiently ample, any sufficiently large multiple $L$ of $L_{1}$ satisfies the property (i): that is, for generic $u \in|L|$ and generic $\alpha \in H^{0}\left(K_{S_{u}}\right)$, the map

$$
\bar{\nabla}^{S}(\alpha): H^{1}\left(T_{S_{u}}\right) \longrightarrow H^{1}\left(\Omega_{S_{u}}\right)_{0}
$$

is injective.

Proof. It is known from [9] that the composition of this map with the inclusion

$$
H^{1}\left(\Omega_{S_{u}}\right)_{0} \subset H^{1}\left(\Omega_{S_{u}}\right)
$$

is nothing but the multiplication map by $\alpha$ :

$$
H^{1}\left(T_{S_{u}}\right) \longrightarrow H^{1}\left(T_{S_{u}} \otimes K_{S_{u}}\right) \cong H^{1}\left(\Omega_{S_{u}}\right) .
$$

So the transposed map

$$
H^{1}\left(\Omega_{S_{u}}\right)_{0} \longrightarrow\left(H^{1}\left(T_{S_{u}}\right)\right)^{*} \cong H^{1}\left(\Omega_{S_{u}} \otimes K_{S_{u}}\right)
$$

is also the multiplication by $\alpha$, and we have to show that it is surjective for generic $\alpha$. We know from [7] and [10] that for sufficiently ample $L$ and smooth $S \in|L|$, the residues on $S$ of the classes of the 3 -forms $P \omega / s^{2}$ generate $F^{1} H^{2}(S)_{0}$, so that their projections modulo $H^{2,0}(S)$ generate $H^{1}\left(\Omega_{S}\right)_{0}$, where $\omega$ is a generator of $H^{0}\left(K_{X}\right)$, $P$ varies in $H^{0}(2 L)$, and $s \in H^{0}(L)$ is an equation for $S$. Hence we have a surjective map

$$
H^{0}(2 L) \longrightarrow H^{1}\left(\Omega_{S}\right)_{0}
$$

Similarly, considering residues of meromorphic forms $P \omega / s^{3}$, where $P \in H^{0}(3 L)$, we get a surjective map

$$
H^{0}(3 L) \longrightarrow H^{2}\left(\mathcal{O}_{S}\right)
$$

One then shows exactly as in [2] that for $\alpha \in H^{0}(L)$, one has the commutative diagram

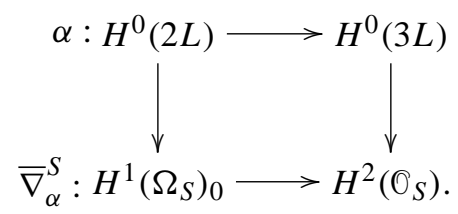

These maps can be obtained as well by looking at the exact sequence

$$
0 \longrightarrow \mathrm{O}_{S}(-L) \longrightarrow \Omega_{X \mid S} \longrightarrow \Omega_{S} \longrightarrow 0
$$


which, by taking the second exterior power and tensoring with $L$, gives

$$
0 \longrightarrow \Omega_{S} \longrightarrow\left(\Omega_{X \mid S}^{2}(L)\right) \longrightarrow K_{S}(L) \longrightarrow 0 .
$$

Using the isomorphism $K_{S}(L) \cong 2 L_{\mid S}$ given by $\omega$ and the fact that the induced map

$$
H^{1}\left(\Omega_{S_{u}}\right) \longrightarrow H^{1}\left(\Omega_{X \mid S}^{2}(L)\right) \cong H^{2}\left(\Omega_{X}^{2}\right)
$$

is equal to $j_{S_{*}}$, we get by the long exact sequence induced by (4.14) the desired map $H^{0}\left(2 L_{\mid S}\right) \rightarrow H^{1}\left(\Omega_{S}\right)_{0}$, with kernel $H^{0}\left(\Omega_{X \mid S}^{2}(L)\right)$.

Tensoring (4.14) by any line bundle $L^{\prime}$, we also get maps

$$
H^{0}\left(2 L+L_{\mid S}^{\prime}\right) \longrightarrow H^{1}\left(\Omega_{S}\left(L^{\prime}\right)\right),
$$

and in particular

$$
H^{0}\left(3 L_{\mid S}\right) \longrightarrow H^{1}\left(\Omega_{S}(L)\right) .
$$

The map (4.11) is then simply obtained by composing the map (4.15) with the map

$$
\delta: H^{1}\left(\Omega_{S}(d)\right) \longrightarrow H^{2}\left(\mathrm{O}_{S}\right)
$$

deduced from the exact sequence (4.13) twisted by $L$. It is then obvious that the following diagram is commutative:

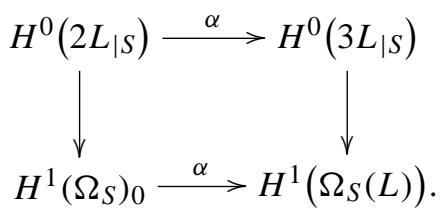

Furthermore, it also follows from the commutativity of diagrams (4.12) and (4.17) that for $\lambda \in H^{1}\left(\Omega_{S}\right)_{0}, \alpha \in H^{0}\left(\mathcal{O}_{S}(L)\right)$, one has

$$
\bar{\nabla}_{\alpha}^{S}(\lambda)=\delta(\alpha \lambda)
$$

In order to show the surjectivity of

$$
\alpha: H^{1}\left(\Omega_{S}\right)_{0} \longrightarrow H^{1}\left(\Omega_{S}(L)\right)
$$

for generic $S$ and $\alpha$, we do the following. Let $L_{1}=\mathscr{O}_{X}(1)$ be sufficiently ample on $X$ and let $\phi_{0}, \ldots, \phi_{3} \in H^{0}\left(L_{1}\right)$ define a map $\phi: X \rightarrow \mathbb{P}^{3}$. For $d$ sufficiently large, let $\Sigma \subset \mathbb{P}^{3}$ be defined by $\sigma \in H^{0}\left(\mathcal{O}_{\mathbb{P}^{3}}(d)\right)$ and let $S=\phi^{-1}(\Sigma)$ be defined by $s=\phi^{*}(\sigma) \in H^{0}\left(\mathcal{O}_{X}(d)\right)$. Let $R \in\left|\mathcal{O}_{X}(4)\right|$ be the ramification locus of $\phi$. For $\Sigma$ we have the exact sequences analogous to (4.14):

$$
0 \longrightarrow \Omega_{\Sigma}(k) \longrightarrow \Omega_{\mathbb{\mathbb { P }}{ }^{3} \mid \Sigma}^{2}(d+k) \longrightarrow K_{\Sigma}(d+k) \longrightarrow 0,
$$


which can be pulled back to $S$ and which give rise to maps (taking into account the isomorphism $K_{\Sigma} \cong O_{\Sigma}(d-4)$ )

$$
H^{0}\left(\mathcal{O}_{S}(2 d-4+k)\right) \longrightarrow H^{1}\left(\phi^{*} \Omega_{\Sigma}(k)\right)
$$

We have the following lemma.

Lemma 11. The diagram

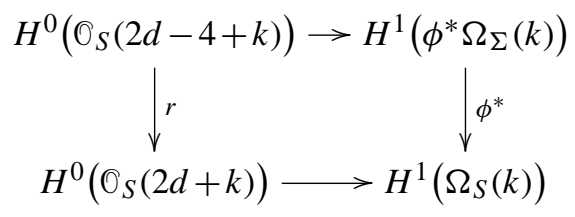

is commutative for an adequate choice of equation $r \in H^{0}\left(\mathrm{O}_{X}(4)\right)$ for $R$.

This follows easily from the fact that the composite

$$
T_{X} \stackrel{\phi_{*}}{\longrightarrow} \phi^{*}\left(T_{\mathbb{P} 3}\right) \cong \phi^{*}\left(\Omega_{\mathbb{P} 3}^{2}(4)\right) \stackrel{\phi_{*}}{\longrightarrow} \Omega_{X}^{2}(4) \cong T_{X}(4)
$$

is the multiplication by $r$, where the choice of $r$ is determined by the isomorphisms $K_{X} \cong \mathcal{O}_{X}$ and $K_{\mathbb{P} 3} \cong \mathcal{O}_{\mathbb{P} 3}(-4)$.

As a consequence of Lemma 11, we get the following.

LEMma 12. Let $d$ be sufficiently large, and let $\Sigma, t \in H^{0}\left(\mathcal{O}_{\Sigma}(d-4)\right)$ satisfy the following condition: the multiplication map

$$
t: H^{1}\left(\Omega_{\Sigma}(-4)\right) \longrightarrow H^{1}\left(\Omega_{\Sigma}(d-8)\right)
$$

is surjective. Then the multiplication map

$$
\phi^{*} t: H^{1}\left(\Omega_{S}\right)_{0} \longrightarrow H^{1}\left(\Omega_{S}(d-4)\right)
$$

satisfies that $\operatorname{Im} \phi^{*} t$ contains $r H^{1}\left(\Omega_{S}(d-8)\right)$.

Proof. From Lemma 11 we conclude that the image of the map

$$
\phi^{*}: H^{1}\left(\phi^{*} \Omega_{\Sigma}(d-4)\right) \longrightarrow H^{1}\left(\Omega_{S}(d-4)\right)
$$

contains $r H^{1}\left(\Omega_{S}(d-8)\right)$. Indeed, we have the commutative diagrams

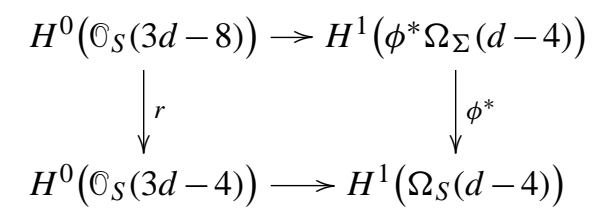


and

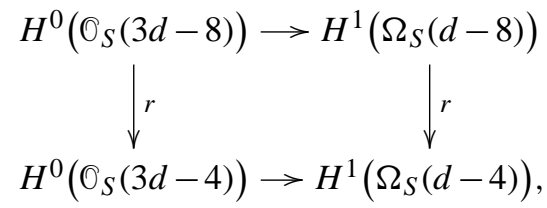

where the surjectivity of the first horizontal map is easy to check.

So it suffices to prove that if $d$ is large enough, the assumption on $\Sigma, t$ implies that the multiplication map

$$
\phi^{*} t: H^{1}\left(\phi^{*} \Omega_{\Sigma}\right)_{0} \longrightarrow H^{1}\left(\phi^{*} \Omega_{\Sigma}(d-4)\right)
$$

is surjective. Now consider the exact sequences

$$
\begin{gathered}
0 \longrightarrow J^{2 d-8} \longrightarrow H^{0}\left(\mathrm{O}_{\Sigma}(2 d-8)\right) \longrightarrow H^{1}\left(\Omega_{\Sigma}(-4)\right) \longrightarrow 0 \\
0 \longrightarrow J^{3 d-12} \longrightarrow H^{0}\left(\mathrm{O}_{\Sigma}(3 d-12)\right) \longrightarrow H^{1}\left(\Omega_{\Sigma}(d-8)\right) \longrightarrow 0
\end{gathered}
$$

constructed above, where $J^{*} \subset H^{0}\left(\mathscr{O}_{\Sigma}(*)\right)$ is the Jacobian ideal of $\Sigma$, that is, the image of $H^{0}\left(\Omega_{\mathbb{p} 3}^{2}(-4+*-d)_{\mid \Sigma}\right)$ under the map induced by (4.19). The hypothesis on $t$ means exactly that

$$
J^{3 d-12}+t H^{0}\left(\mathcal{O}_{\Sigma}(2 d-8)\right)=H^{0}\left(\mathcal{O}_{\Sigma}(3 d-12)\right) .
$$

Now if $d$ is large enough, the multiplication map

$$
H^{0}\left(\mathrm{O}_{S}(4)\right) \otimes \phi^{*} H^{0}\left(\mathbb{O}_{\Sigma}(3 d-12)\right) \longrightarrow H^{0}\left(\mathscr{O}_{S}(3 d-8)\right)
$$

is surjective. It follows that

$$
H^{0}\left(\mathscr{O}_{S}(4)\right) \cdot \phi^{*} J^{3 d-12}+\phi^{*} t H^{0}\left(\mathscr{O}_{S}(2 d-4)\right)=H^{0}\left(\mathcal{O}_{S}(3 d-8)\right) .
$$

Since $H^{0}\left(\mathcal{O}_{S}(4)\right) \cdot \phi^{*} J^{3 d-12}$ vanishes in $H^{1}\left(\phi^{*} \Omega_{\Sigma}(d-4)\right)$ and the map

$$
H^{0}\left(\mathrm{O}_{S}(3 d-8)\right) \longrightarrow H^{1}\left(\phi^{*} \Omega_{\Sigma}(d-4)\right)
$$

is surjective, it follows that

$$
\phi^{*} t: H^{1}\left(\phi^{*} \Omega_{\Sigma}\right)_{0} \longrightarrow H^{1}\left(\phi^{*} \Omega_{\Sigma}(d-4)\right)
$$

is surjective.

We now conclude the proof of Proposition 2. It is quite easy to verify that for generic $\Sigma, t$ the condition of Lemma 12 is satisfied. So we have $(\Sigma, t)$ such that $\operatorname{Im} \phi^{*} t$ contains $r H^{1}\left(\Omega_{S}(d-8)\right)$. We want to conclude that

$$
\phi^{*} t: H^{1}\left(\Omega_{S}\right)_{0} \longrightarrow H^{1}\left(\Omega_{S}(d-4)\right)
$$


is in fact surjective.

Consider the surjective map $H^{0}\left(\mathscr{O}_{S}(3 d-4)\right) \rightarrow H^{1}\left(\Omega_{S}(d-4)\right)$. It has for kernel the space

$$
J_{S}^{3 d-4}=\left\{d s(u), u \in H^{0}\left(T_{X}(2 d-4)_{\mid S}\right)\right\} .
$$

The fact that $\operatorname{Im} \phi^{*} t$ contains $r H^{1}\left(\Omega_{S}(d-8)\right)$ means then that $r H^{0}\left(\mathscr{O}_{S}(3 d-8)\right)_{\mid \phi^{*} t=s=0}$ is contained in $\left.J_{S}^{3 d-4}\right|_{\phi^{*} t=s=0}$. Now let

$$
s_{\epsilon}=s+\epsilon r \phi^{*} t .
$$

We first show that for generic $t, \sigma$, and $\phi$

$$
r H^{1}\left(\Omega_{S_{\epsilon}}(d-8)\right)=H^{1}\left(\Omega_{S_{\epsilon}}(d-4)\right) .
$$

Equivalently, we have to show that the multiplication map

$$
r: H^{1}\left(T_{S_{\epsilon}}(4)\right) \rightarrow H^{1}\left(T_{S_{\epsilon}}(8)\right)
$$

is injective. Looking at the exact sequences

$$
\begin{aligned}
& 0 \longrightarrow T_{S_{\epsilon}}(4) \longrightarrow T_{X}(4)_{\mid S_{\epsilon}} \longrightarrow \mathcal{O}_{S_{\epsilon}}(d+4) \longrightarrow 0 \\
& 0 \longrightarrow T_{S_{\epsilon}}(8) \longrightarrow T_{X}(8)_{\mid S_{\epsilon}} \longrightarrow \mathcal{O}_{S_{\epsilon}}(d+8) \longrightarrow 0
\end{aligned}
$$

and using the fact that $O_{X}(1)$ is sufficiently ample, we find that the kernel of the map (4.25) identifies to the set

$$
\left\{u \in H^{0}\left(T_{X}(8)_{\mid R}\right), d s_{\epsilon}(u)_{\mid r=s_{\epsilon}=0}=0\right\} .
$$

Now we have the equality

$$
d s_{\epsilon}(u)_{\mid r=s_{\epsilon}=0}=d s(u)_{\mid r=s_{\epsilon}=0}+\epsilon t d r(u)_{\mid r=s_{\epsilon}=0},
$$

where the curves $\{r=s=0\}$ and $\left\{r=s_{\epsilon}=0\right\}$ coincide. (Notice that all these derivatives only make sense when restricted to the vanishing locus of the considered equation.) Now clearly for sufficiently large $d$, general $t$, and any $u$ in the fixed vector space $H^{0}\left(T_{X}(8)_{\mid R}\right)$, the right-hand side vanishes if and only if

$$
d s(u)_{\mid r=s_{\epsilon}=0}=0 \quad \text { and } \quad d r(u)_{\mid r=s_{\epsilon}=0}=0 .
$$

But if $\phi$ is generic, the surface $R$ is reduced, and the second condition means that $u$ is tangent to it. Then clearly there is at most for each such $u$ a one-dimensional family of curves $\{r=s=0\}$ on the surface $R$ which are tangent to $u$; that is, $s$ satisfies the first condition. Since $u$ varies in the fixed subspace of $H^{0}\left(T_{X}(8)_{\mid R}\right)$ of elements tangent to $R$, it follows that for $d$ large enough and generic $\sigma$, the two conditions above imply that $u=0$, so that the map (4.25) is injective. 
This means, as above, that we have

$$
J_{S_{\epsilon}}^{3 d-4}{ }_{\mid r=s_{\epsilon}=0}=H^{0}\left(\mathcal{O}_{S_{\epsilon}}(3 d-4)\right)_{\mid r=s_{\epsilon}=0},
$$

so that, in particular,

$$
J_{S_{\epsilon}}^{3 d-4}{ }_{\mid \phi * t=r=s_{\epsilon}=0}=H^{0}\left(\mathcal{O}_{S_{\epsilon}}(3 d-4)\right)_{\mid \phi^{*} t=r=s_{\epsilon}=0} .
$$

But the curve defined by $s=\phi^{*} t=0$ is equal to the curve defined by $s_{\epsilon}=\phi^{*} t=0$; the restriction map

$$
H^{0}\left(T_{X}(2 d-4)\right) \longrightarrow H^{0}\left(T_{X}(2 d-4)_{\mid S}\right)
$$

is surjective, and for $u \in H^{0}\left(T_{X}(2 d-4)\right)$, we have

$$
d s_{\epsilon}(u)_{\mid r=s=\phi^{*} t=0}=d s(u)_{\mid r=s=t=0} .
$$

It follows that we have as well

$$
J_{S}^{3 d-4}{ }_{\mid \phi * t=r=s=0}=H^{0}\left(\mathcal{O}_{S}(3 d-4)\right)_{\mid \phi^{*} t=r=s=0} .
$$

Since $J_{S}^{3 d-4}{ }_{\mid \phi * t=s=0}$ contains $r H^{0}\left(\mathscr{O}_{S}(3 d-8)\right)_{\mid \phi^{*} t=s=0}$, this implies that

$$
J_{S}^{3 d-4}{ }_{\mid \phi * t=s=0}=H^{0}\left(\mathcal{O}_{S}(3 d-4)\right)_{\mid \phi^{*} t=s=0},
$$

which is equivalent to the fact that $\phi^{*} t: H^{1}\left(\Omega_{S}\right)_{0} \rightarrow H^{1}\left(\Omega_{S}(d-4)\right)$ is surjective. Finally, it is easy to check that for generic $t^{\prime} \in H^{0}\left(\mathscr{O}_{\Sigma}(4)\right)$, the multiplication map

$$
\phi^{*} t^{\prime}: H^{1}\left(\Omega_{S}(d-4)\right) \longrightarrow H^{1}\left(\Omega_{S}(d)\right)
$$

is surjective, so we have proved that for generic $t \in H^{0}\left(\mathscr{O}_{\Sigma}(d)\right)$ the multiplication map

$$
\phi^{*} t: H^{1}\left(\Omega_{S}\right)_{0} \longrightarrow H^{1}\left(\Omega_{S}(d)\right)
$$

is surjective. Thus Proposition 2 is proved.

It remains now to check condition (ii) in Proposition 1.

Proposition 3. Let $L_{1}$ be ample on the Calabi-Yau threefold $X$. Then for any sufficiently large multiple $L$ of $L_{1}$ and any generic $S \in|L|, \alpha \in H^{0}\left(L_{\mid S}\right)$, and $\lambda \in$ $H^{1}\left(\Omega_{S}\right)_{0}$ such that $\bar{\nabla}^{S}(\lambda)(\alpha)=0$ in $H^{2}\left(\mathcal{O}_{S}\right)$, we have that

$$
\operatorname{Ker}\left(\bar{\nabla}^{S}(\lambda): H^{0}\left(L_{\mid S}\right) \longrightarrow H^{2}\left(\mathrm{O}_{S}\right)\right)
$$

is generated by $\alpha$.

We follow this strategy: We again consider a generic map $\phi: X \rightarrow \mathbb{P}^{3}$, with $L_{1}=\mathscr{O}_{X}(1)=\phi^{*}\left(\mathbb{O}_{\mathbb{P} 3}(1)\right)$ sufficiently ample, and surfaces $S=\phi^{-1}(\Sigma)$ for generic 
$\Sigma \subset \mathbb{P}^{3}$ of degree $d$ sufficiently large. So $S=V(s), \Sigma=V(\sigma)$ with $s=\phi^{*}(\sigma)$.

Next let $t \in H^{0}\left(\mathbb{O}_{\Sigma}(d)\right)$ be generic. Then for $\alpha=\phi^{*} t$, we know that $\bar{\nabla}_{\alpha}^{S}$ : $H^{1}\left(\Omega_{S}\right)_{0} \rightarrow H^{2}\left(\mathcal{O}_{S}\right)$ is surjective, so the set of $\lambda \in H^{1}\left(\Omega_{S}\right)_{0}$ such that $\bar{\nabla}^{S}(\lambda)(\alpha)=0$ in $H^{2}\left(\mathbb{O}_{S}\right)$, which is equal to $\operatorname{Ker} \bar{\nabla}_{\alpha}^{S}$, has the minimal generic dimension. Hence it suffices to prove Proposition 3 for such $(S, \alpha)$.

First, we show that for generic $\lambda \in H^{1}\left(\phi^{*} \Omega_{\Sigma}\right)_{0}$ such that $\bar{\nabla}^{S}(\lambda)(\alpha)=0$ in $H^{2}\left(\mathscr{O}_{S}\right)$, that is, $\lambda \in \operatorname{Ker} \bar{\nabla}_{\alpha}^{S}$, the kernel of

$$
\bar{\nabla}^{S}(\lambda): H^{0}\left(\mathrm{O}_{S}(d)\right) \longrightarrow H^{2}\left(\mathrm{O}_{S}\right)
$$

is generated by $\alpha$ and

$$
\tilde{J}_{\Sigma}:=\operatorname{Ker}\left(H^{0}\left(\mathcal{O}_{S}(d)\right) \longrightarrow H^{1}\left(T_{\Sigma}\right)\right)=\operatorname{Im}\left(H^{0}\left(\phi^{*}\left(T_{\mathbb{P}^{3}}\right)\right) \longrightarrow H^{0}\left(\mathcal{O}_{S}(d)\right)\right) .
$$

Then we conclude that for generic $\lambda \in \operatorname{Ker} \bar{\nabla}_{\alpha}^{S}$, the kernel of

$$
\bar{\nabla}^{S}(\lambda): H^{0}\left(\mathrm{O}_{S}(d)\right) \longrightarrow H^{2}\left(\mathrm{O}_{S}\right)
$$

is generated by $\alpha$, by showing that the set of quadrics $q_{\lambda}$ on $\mathbb{P}\left(H^{0}\left(\mathbb{O}_{S}(d)\right)\right)$ for $\lambda \in \operatorname{Ker} \bar{\nabla}_{\alpha}^{S}$ has no base point on $\mathbb{P}\left(\tilde{J}_{\Sigma}\right)$.

Let us introduce the notation $R_{\sigma}=\mathbb{C}\left[X_{0}, \ldots, X_{3}\right] / J_{\sigma}$, where $J_{\sigma}$ is the ideal generated by the partial derivatives $\frac{\partial \sigma}{\partial X_{i}}$. Using (4.19), we get isomorphisms

$$
R_{\sigma}^{2 d-4} \cong H^{1}\left(\Omega_{\Sigma}\right)_{0}, \quad R_{\sigma}^{2 d-4+k} \cong H^{1}\left(\Omega_{\Sigma}(k)\right),
$$

for any integer $k \neq 0$. Furthermore, $R_{\sigma}$ is Gorenstein: we have $R_{\sigma}^{4 d-8} \cong \mathbb{C}$, and the pairings

$$
R_{\sigma}^{2 d-4-k} \times R_{\sigma}^{2 d-4+k} \longrightarrow R_{\sigma}^{4 d-8}
$$

are perfect. We first show the following.

Proposition 4. Assume that $\mathrm{O}_{X}(1)$ is sufficiently ample, that $\phi$ is generic, and that $d$ is sufficiently large. Let $t \in H^{0}\left(\mathcal{O}_{\Sigma}(d)\right)$ be generic and assume that there exist $\lambda_{1} \in R_{\sigma}^{2 d-8} \cong H^{1}\left(\Omega_{\Sigma}(-4)\right), A \in H^{0}\left(\mathbb{O}_{\Sigma}(2)\right)$ satisfying the following properties:

(a) Ker $\lambda_{1}: R_{\sigma}^{d} \rightarrow R_{\sigma}^{3 d-8} \cong\left(R_{\sigma}^{d}\right)^{*}$ is generated by the image $\bar{t}$ of $t$ in $R_{\sigma}^{d}$;

(b) $A \lambda_{1}: R_{\sigma}^{d-1} \rightarrow R_{\sigma}^{3 d-7} \cong\left(R_{\sigma}^{d-1}\right)^{*}$ is an isomorphism;

(c) $A^{2} \lambda_{1}: R_{\sigma}^{d-2} \rightarrow R_{\sigma}^{3 d-6} \cong\left(R_{\sigma}^{d-2}\right)^{*}$ is an isomorphism;

(d) $A^{3} \lambda_{1}: R_{\sigma}^{d-3} \rightarrow R_{\sigma}^{3 d-5} \cong\left(R_{\sigma}^{d-3}\right)^{*}$ is an isomorphism;

(e) $A^{4} \lambda_{1}: R_{\sigma}^{d-4} \rightarrow R_{\sigma}^{3 d-4} \cong\left(R_{\sigma}^{d-4}\right)^{*}$ is an isomorphism.

Then for $\psi \in H^{0}\left(\mathscr{O}_{X}(4)\right)$ generic and $Q \in H^{0}\left(\mathscr{O}_{X}(2)\right)$ generic, the element

$$
\lambda=\psi \phi^{*}\left(\lambda_{1}\right)+Q \phi^{*}\left(A \lambda_{1}\right)+\phi^{*}\left(A^{2} \lambda_{1}\right)
$$


of $H^{1}\left(\phi^{*} \Omega_{\Sigma}\right)_{0}$ satisfies that $\bar{\nabla}^{S}(\lambda)(\alpha)=0$ in $H^{2}\left(\mathscr{O}_{S}\right)$, where $\alpha=\phi^{*}(t)$, and the kernel of

$$
\bar{\nabla}^{S}(\lambda): H^{0}\left(\mathrm{O}_{S}(d)\right) \longrightarrow H^{2}\left(\mathbb{O}_{S}\right)
$$

is generated by $\alpha$ and $\tilde{J}_{\Sigma}$.

It is clear that $\alpha$ is in the kernel of $\bar{\nabla}^{S}(\lambda)$, since we have $\lambda=f \phi^{*}\left(\lambda_{1}\right)$, where $\lambda_{1} \in H^{1}\left(\Omega_{\Sigma}(-4)\right)$ satisfies $t \lambda_{1}=0$ in $H^{1}\left(\Omega_{\Sigma}(d-4)\right)$. This implies that $\alpha \lambda=0$ in $H^{1}\left(\Omega_{S}(d)\right)$ and a fortiori $\bar{\nabla}^{S}(\lambda)(\alpha)=0$ in $H^{2}\left(\mathscr{O}_{S}\right)$, since we have by (4.18)

$$
\bar{\nabla}^{S}(\lambda)(\alpha)=\delta(\alpha \lambda)
$$

Also $\tilde{J}_{\Sigma}$ is contained in the kernel of $\bar{\nabla}^{S}(\lambda)$. Indeed, since $\lambda \in H^{1}\left(\phi^{*} \Omega_{\Sigma}\right)_{0}$, the map

$$
\bar{\nabla}^{S}(\lambda): H^{1}\left(T_{S}\right) \longrightarrow H^{2}\left(\mathrm{O}_{S}\right),
$$

which is given by interior product, clearly factors through $H^{1}\left(\phi^{*}\left(T_{\Sigma}\right)\right)$. Let us first prove the following lemma.

Lemma 13. Let $\lambda^{\prime}=A^{2} \lambda_{1} \in R_{\sigma}^{2 d-4}$. Assumptions $(a), \ldots,(e)$ on $\lambda_{1}$, A imply the following:

(i) $\lambda^{\prime}: R_{\sigma}^{d-2} \rightarrow R_{\sigma}^{3 d-6} \cong\left(R_{\sigma}^{d-2}\right)^{*}$ is an isomorphism;

(ii) $A \lambda_{1}:\left(\operatorname{Ker} \lambda^{\prime}\right)^{d-1} \rightarrow\left(\text { Coker } \lambda^{\prime}\right)^{3 d-7}$ is an isomorphism;

(iii) $\lambda_{1}:\left(\operatorname{Ker} \lambda^{\prime}\right)^{d} \rightarrow\left(\text { Coker } \lambda^{\prime}\right)^{3 d-8}$ has its kernel generated by $t$.

Here we denote by $\left(\operatorname{Ker} \lambda^{\prime}\right)^{*}$ (resp., $\left.\left(\operatorname{Coker} \lambda^{\prime}\right)^{*}\right)$ the kernel of the multiplication by $\lambda^{\prime}: R_{\sigma}^{*} \rightarrow R_{\sigma}^{2 d-4+*}$ (resp., the cokernel of the multiplication by $\lambda^{\prime}: R_{\sigma}^{*-2 d+4} \rightarrow R_{\sigma}^{*}$ ).

Proof. (i) is assumption (c).

(ii) Let $u \in\left(\operatorname{Ker} \lambda^{\prime}\right)^{d-1}$ and assume $A \lambda_{1} u=0$ in $\left(\operatorname{Coker} \lambda^{\prime}\right)^{3 d-7}$. This means that $A \lambda_{1} u=A^{2} \lambda_{1} v$ in $R_{\sigma}^{3 d-7}$ for some $v \in R_{\sigma}^{d-3}$. By assumption (b), it follows that $u=A v$. Then $A^{2} \lambda_{1} u=0$ implies that $A^{3} \lambda_{1} v=0$; by (d), $v=0$, so $u=0$.

We prove (iii) in the same way.

In order to prove Proposition 4, we first study the map

$$
\bar{\mu}_{\lambda^{\prime}}: H^{1}\left(\phi^{*}\left(T_{\Sigma}\right)\right) \longrightarrow H^{1}\left(\phi^{*} \Omega_{\Sigma}(d)\right),
$$

which is the factorization of the multiplication map by $\phi^{*}\left(\lambda^{\prime}\right) \in H^{1}\left(\phi^{*} \Omega_{\Sigma}\right)$ :

$$
\mu_{\lambda^{\prime}}: H^{0}\left(\mathcal{O}_{S}(d)\right) \longrightarrow H^{1}\left(\phi^{*} \Omega_{\Sigma}(d)\right),
$$

using the surjective map

$$
H^{0}\left(\mathcal{O}_{S}(d)\right) \longrightarrow H^{1}\left(\phi^{*}\left(T_{\Sigma}\right)\right) .
$$

(We use the fact that $H^{1}\left(\phi^{*}\left(T_{\mathbb{P}^{3}}\right)_{\mid S}\right)=0$.) Notice that from (4.18), the composition of $\bar{\mu}_{\lambda^{\prime}}$ with the map $\delta: H^{1}\left(\phi^{*} \Omega_{\Sigma}\right) \rightarrow H^{2}\left(\mathscr{O}_{S}\right)$ of (4.16) is equal to the factorization through $H^{1}\left(\phi^{*}\left(T_{\Sigma}\right)\right)$ of $\bar{\nabla}^{S}\left(\phi^{*}\left(\lambda^{\prime}\right)\right)_{\mid H^{0}\left(\mathscr{O}_{S}(d)\right)}$. We have the following lemma. 
Lemma 14. Let $K=\left(H^{0}\left(\mathcal{O}_{X}(1)\right) / H^{0}\left(\mathscr{O}_{\mathbb{P} 3}(1)\right)\right)^{*}$. Choose a splitting

$$
H^{0}\left(\mathcal{O}_{X}(1)\right) \cong K^{*} \oplus H^{0}\left(\mathcal{O}_{\mathbb{P} 3}(1)\right) ;
$$

then $\operatorname{Ker} \bar{\mu}_{\lambda^{\prime}}$ is naturally isomorphic to $\left(\operatorname{Ker} \lambda^{\prime}\right)^{d} \oplus K^{*} \otimes\left(\operatorname{Ker} \lambda^{\prime}\right)^{d-1}$, and Coker $\bar{\mu}_{\lambda^{\prime}}$ is naturally isomorphic to $\left(\text { Coker } \lambda^{\prime}\right)^{3 d-8} \oplus K \otimes\left(\text { Coker } \lambda^{\prime}\right)^{3 d-7}$.

Notice that the map from $\left(\operatorname{Ker} \lambda^{\prime}\right)^{d} \oplus K^{*} \otimes\left(\operatorname{Ker} \lambda^{\prime}\right)^{d-1}$ to $\operatorname{Ker} \bar{\mu}_{\lambda^{\prime}}$ is the natural one: indeed, $\left(\operatorname{Ker} \lambda^{\prime}\right)^{d}$ identifies to the kernel of $\bar{\mu}_{\lambda^{\prime} \mid \phi^{*}\left(H^{1}\left(T_{\Sigma}\right)\right)}$ while $\left(\operatorname{Ker} \lambda^{\prime}\right)^{d-1}$ identifies to the kernel of the analogous map

$$
H^{1}\left(\phi^{*}\left(T_{\Sigma}\right)(-1)\right) \longrightarrow H^{1}\left(\phi^{*} \Omega_{\Sigma}(d-1)\right)
$$

restricted to $\phi^{*}\left(H^{1}\left(T_{\Sigma}(-1)\right)\right)$.

Notice also that both statements are dual to each other: indeed, the map $\bar{\mu}_{\lambda^{\prime}}$ is symmetric with respect to the Serre duality isomorphism

$$
H^{1}\left(\phi^{*}\left(T_{\Sigma}\right)\right) \cong\left(H^{1}\left(\phi^{*} \Omega_{\Sigma}(d)\right)\right)^{*} ;
$$

so $\left(\operatorname{Ker} \lambda^{\prime}\right)^{d}$ is dual to $\left(\operatorname{Coker} \lambda^{\prime}\right)^{3 d-8}$ and $\left(\operatorname{Ker} \lambda^{\prime}\right)^{d-1}$ is dual to $\left(\operatorname{Coker} \lambda^{\prime}\right)^{3 d-7}$ by the pairings (4.28).

So it suffices to prove the second statement, and for this we can replace $\bar{\mu}_{\lambda^{\prime}}$ by $\mu_{\lambda^{\prime}}$. To prove it we first prove the following.

Lemma 15. Let $\mathscr{E}$ be the vector bundle $\phi_{*} \mathrm{O}_{X}(2)$ on $\mathbb{P}^{3}$, and let $\mathscr{Y}$ be the cokernel of the natural map

$$
H^{0}\left(\mathscr{O}_{X}(2)\right) \otimes \mathbb{O}_{\mathbb{P}^{3}} \longrightarrow \mathscr{E} ;
$$

then the splitting (4.29) gives an isomorphism

$$
\mathscr{K} \cong \mathbb{O}_{\mathbb{P} 3}(-2) \oplus\left(K \otimes \mathbb{O}_{\mathbb{P} 3}(-1)\right) .
$$

Proof. Let $\Gamma \subset X \times \mathbb{P}^{3}$ be the graph of $\phi$. Then

$$
\mathscr{K}=R^{1} p r_{2 *}\left(\mathscr{I}_{\Gamma} \otimes p r_{1}^{*}\left(\mathscr{O}_{X}(2)\right)\right) .
$$

Now if $Q$ is defined by the exact sequence

$$
0 \longrightarrow Q \longrightarrow H^{0}\left(\mathbb{O}_{\mathbb{P}^{3}}(1)\right) \otimes \mathbb{O}_{\mathbb{P}^{3}} \longrightarrow \mathbb{O}_{\mathbb{P}^{3}}(1) \longrightarrow 0,
$$

$\mathscr{I}_{\Gamma}$ has the resolution

$$
\begin{aligned}
0 & \longrightarrow \bigwedge^{3}\left(p r_{2}^{*} Q \otimes p r_{1}^{*}\left(\mathrm{O}_{X}(-1)\right)\right) \longrightarrow \bigwedge^{2}\left(p r_{2}^{*} Q \otimes p r_{1}^{*}\left(\mathrm{O}_{X}(-1)\right)\right) \\
& \longrightarrow p r_{2}^{*} Q \otimes p r_{1}^{*}\left(\mathcal{O}_{X}(-1)\right) \longrightarrow \Phi_{\Gamma} \longrightarrow 0
\end{aligned}
$$

One concludes from this that $\mathscr{K}$ is isomorphic to

$$
\operatorname{Ker} \beta: \bigwedge^{3} Q \otimes H^{3}\left(\mathrm{O}_{X}(-1)\right) \longrightarrow \bigwedge^{2} Q \otimes H^{3}\left(\mathbb{O}_{X}\right) .
$$


Now the dual of the map $\beta$ is simply the natural map

$$
\alpha: Q \otimes \mathbb{O}_{\mathbb{P} 3}(1) \longrightarrow H^{0}\left(\mathscr{O}_{X}(1)\right) \otimes \mathscr{O}_{\mathbb{P} 3}(1),
$$

from which it follows easily that

$$
\mathscr{K}=(\operatorname{Coker} \alpha)^{*} \cong \mathbb{O}_{\mathbb{P}^{3}}(-2) \oplus\left(K \otimes \mathcal{O}_{\mathbb{P}^{3}}(-1)\right) .
$$

Tensorizing the exact sequence

$$
H^{0}\left(\mathcal{O}_{X}(2)\right) \otimes \mathbb{O}_{\mathbb{P} 3} \longrightarrow \mathscr{E} \longrightarrow \mathscr{K} \longrightarrow 0
$$

with $0_{\Sigma}(d-2)$, we deduce easily from Lemma 15 the following.

COROLlary 4. The splitting (4.29) gives an isomorphism

$$
\frac{H^{0}\left(\mathcal{O}_{S}(d)\right)}{H^{0}\left(\mathcal{O}_{X}(2)\right) H^{0}\left(\mathcal{O}_{\Sigma}(d-2)\right)} \cong H^{0}\left(\mathcal{O}_{\Sigma}(d-4)\right) \oplus\left(K \otimes H^{0}\left(\mathcal{O}_{\Sigma}(d-3)\right)\right) .
$$

Similarly, tensorizing the exact sequence (4.31) with $\Omega_{\Sigma}(d-2)$ and using Lemma 15 , we easily get the following.

COROLlary 5. The splitting (4.29) gives an isomorphism

$$
\frac{H^{1}\left(\phi^{*} \Omega_{\Sigma}(d)\right)}{H^{0}\left(\mathcal{O}_{X}(2)\right) \phi^{*} H^{1}\left(\Omega_{\Sigma}(d-2)\right)} \cong H^{1}\left(\Omega_{\Sigma}(d-4)\right) \oplus K \otimes H^{1}\left(\Omega_{\Sigma}(d-3)\right) .
$$

Proof of Lemma 14. By assumption (i) on $\lambda^{\prime}$, it follows that $\operatorname{Im} \mu_{\lambda^{\prime}}$ contains $H^{0}\left(\mathcal{O}_{X}(2) \phi^{*}\left(H^{1}\left(\Omega_{\Sigma}(d-2)\right)\right.\right.$, since it means that the map $\lambda^{\prime}: H^{1}\left(T_{\Sigma}(-2)\right) \rightarrow$ $H^{1}\left(\Omega_{\Sigma}(d-2)\right)$ is surjective.

So it suffices to study the cokernel of the induced map

$$
\rho_{\lambda^{\prime}}: \frac{H^{0}\left(\mathcal{O}_{S}(d)\right)}{H^{0}\left(\mathcal{O}_{X}(2)\right) H^{0}\left(\mathscr{O}_{\Sigma}(d-2)\right)} \longrightarrow \frac{H^{1}\left(\phi^{*} \Omega_{\Sigma}(d)\right)}{H^{0}\left(\mathcal{O}_{X}(2)\right) \phi^{*} H^{1}\left(\Omega_{\Sigma}(d-2)\right)} .
$$

But applying Corollaries 4 and $5, \rho_{\lambda^{\prime}}$ gives a map

$$
H^{0}\left(\mathcal{O}_{\Sigma}(d-4)\right) \oplus\left(K \otimes H^{0}\left(\mathcal{O}_{\Sigma}(d-3)\right)\right) \longrightarrow H^{1}\left(\Omega_{\Sigma}(d-4)\right) \oplus\left(K \otimes H^{1}\left(\Omega_{\Sigma}(d-3)\right)\right) .
$$

This last map is now easily seen to be the direct sum of the multiplication map by $\lambda^{\prime} \in H^{1}\left(\Omega_{\Sigma}\right)_{0}$, from which we conclude that

$$
\text { Coker } \mu_{\lambda^{\prime}} \cong \operatorname{Coker} \rho_{\lambda^{\prime}} \cong\left(\operatorname{Coker} \lambda^{\prime}\right)^{3 d-8} \oplus\left(K \otimes\left(\operatorname{Coker} \lambda^{\prime}\right)^{3 d-7}\right),
$$

using the isomorphisms

$$
H^{1}\left(\Omega_{\Sigma}(d-4)\right) \cong R_{\sigma}^{3 d-8}, \quad H^{1}\left(\Omega_{\Sigma}(d-3)\right) \cong R_{\sigma}^{3 d-7},
$$

of (4.27). 
Next for $Q \in H^{0}\left(\mathscr{O}_{X}(2)\right)$, let $\lambda_{2}=Q \phi^{*}\left(A \lambda_{1}\right) \in H^{1}\left(\phi^{*} \Omega_{\Sigma}\right)_{0}$. Again, the multiplication map

$$
\mu_{\lambda_{2}}: H^{0}\left(\mathcal{O}_{S}(d)\right) \longrightarrow H^{1}\left(\phi^{*} \Omega_{\Sigma}(d)\right)
$$

induces a symmetric map

$$
H^{1}\left(\phi^{*}\left(T_{\Sigma}\right)\right) \longrightarrow H^{1}\left(\phi^{*} \Omega_{\Sigma}(d)\right),
$$

and hence a symmetric map

$$
\bar{\mu}_{\lambda_{2}}: \phi^{*}\left(H^{1}\left(T_{\Sigma}\right)\right) \oplus\left(K^{*} \otimes \phi^{*}\left(H^{1}\left(T_{\Sigma}(-1)\right)\right)\right) \longrightarrow \frac{H^{1}\left(\phi^{*} \Omega_{\Sigma}(d)\right)}{H^{0}\left(\mathcal{O}_{X}(2)\right) H^{1}\left(\phi^{*} \Omega_{\Sigma}(d-2)\right)},
$$

that is, by Lemma 14 a map

$$
\bar{\mu}_{\lambda_{2}}: R_{\sigma}^{d} \oplus\left(K^{*} \otimes R_{\sigma}^{d-1}\right) \longrightarrow R_{\sigma}^{3 d-8} \oplus\left(K \otimes R_{\sigma}^{3 d-7}\right) .
$$

We have the following lemma.

Lemma 16. The map $\bar{\mu}_{\lambda_{2}}$ vanishes on $R_{\sigma}^{d}$, and on $K^{*} \otimes R_{\sigma}^{d-1}$ it is computed as follows: There is a natural map

$$
\Psi: H^{0}\left(\mathrm{O}_{X}(2)\right) \longrightarrow \operatorname{Hom}\left(K^{*}, K\right)
$$

such that

$$
\bar{\mu}_{\lambda_{2}}: K^{*} \otimes R_{\sigma}^{d-1} \longrightarrow K \otimes R_{\sigma}^{3 d-7}
$$

is equal to $\Psi(Q) \otimes A \lambda_{1}$.

Proof. The first statement is obvious, since $\mu_{\lambda_{2}}\left(\phi^{*}\left(H^{0}\left(\mathscr{O}_{\Sigma}(d)\right)\right)\right)$ is contained in $H^{0}\left(\mathcal{O}_{X}(2)\right) \cdot \phi^{*}\left(H^{1}\left(\Omega_{\Sigma}(d-2)\right)\right)$. As for the second one, consider the commutative diagram

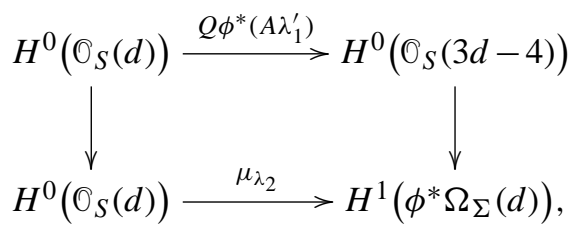

where $\lambda_{1}^{\prime}$ is any lifting of $\lambda_{1}$ in $H^{0}\left(0_{\Sigma}(2 d-8)\right)$; the second vertical map was defined in (4.20). The commutative diagram shows that it suffices to prove more generally the following lemma: Consider the multiplication map

$$
Q \phi^{*} P: \frac{H^{0}\left(\mathcal{O}_{X}(d)\right)}{H^{0}\left(\mathcal{O}_{\mathbb{P}}(2)\right) H^{0}\left(\mathscr{O}_{X}(d-2)\right)} \longrightarrow \frac{H^{0}\left(\mathscr{O}_{X}(d+k+2)\right)}{H^{0}\left(\mathscr{O}_{\mathbb{P}}(2)\right) H^{0}\left(\mathcal{O}_{X}(d+k)\right)}
$$


for any $P \in H^{0}\left(\mathcal{O}_{\mathbb{P} 3}(k)\right)$. Using the isomorphism deduced from Lemma 15,

$$
\frac{H^{0}\left(\mathcal{O}_{X}(d+k+2)\right)}{H^{0}\left(\mathbb{O}_{\mathbb{P}^{3}}(2)\right) H^{0}\left(\mathcal{O}_{X}(d+k)\right)} \cong H^{0}\left(\mathscr{O}_{\mathbb{P}^{3}}(d-2+k)\right) \oplus\left(K \otimes H^{0}\left(\mathscr{O}_{\mathbb{P}^{3}}(d-1+k)\right)\right),
$$

$Q \phi^{*} P$ induces a map

$$
K^{*} \otimes H^{0}\left(\mathcal{O}_{\mathbb{P}^{3}}(d-1)\right) \longrightarrow K \otimes H^{0}\left(\mathcal{O}_{\mathbb{P}^{3}}(d-1+k)\right) ;
$$

then we have the following.

LEMMA 17. There is a natural map

$$
\Psi: H^{0}\left(\mathrm{O}_{X}(2)\right) \longrightarrow \operatorname{Hom}\left(K^{*}, K\right)
$$

such that this map is equal to $\Psi(Q) \otimes P$.

Proof. We construct the map $\Psi$ as follows: Let $\mathscr{L}$ be the cokernel of the natural map

$$
H^{0}\left(\mathrm{O}_{X}(1)\right) \otimes \mathrm{O}_{\mathbb{P} 3} \longrightarrow \phi_{*} \mathrm{O}_{X}(1)
$$

Using the equality

$$
\mathscr{L}=R_{1} p r_{2 *}\left(\mathscr{\Phi}_{\Gamma} \otimes p r_{1}^{*} \mathscr{O}_{X}(1)\right),
$$

where the notation is as in the proof of Lemma 15, and the resolution (4.30), we find that $\mathscr{L}$ is isomorphic to the dual of the cokernel of the natural map

$$
Q(1) \otimes H^{0}\left(\mathscr{O}_{X}(1)\right) \longrightarrow H^{0}\left(\mathcal{O}_{X}(2)\right) \otimes \mathbb{O}_{\mathbb{P}}(1) .
$$

In particular, there is a natural inclusion of $\mathscr{L}$ in $H^{0}\left(\mathscr{O}_{X}(2)\right)^{*} \otimes \mathscr{O}_{\mathbb{p} 3}(-1)$. Tensorizing by $\mathcal{O}_{\mathbb{P}^{3}}(1)$ and taking global sections, we get a map

$$
\chi: H^{0}\left(\mathscr{O}_{X}(2)\right) \longrightarrow\left(H^{0}\left(\mathcal{O}_{X}(2)\right)\right)^{*}
$$

whose image is the set of linear forms vanishing on $H^{0}\left(\mathcal{O}_{\mathbb{P} 3}(1)\right) \cdot H^{0}\left(\mathcal{O}_{X}(1)\right)$. Recalling that $K^{*}=H^{0}\left(\mathscr{O}_{X}(1)\right) / H^{0}\left(\mathscr{O}_{\mathbb{P} 3}(1)\right)$, such a linear form $\chi(Q)$ obviously induces a symmetric bilinear form on $K^{*}$ and, hence, a map $\Psi(Q): K^{*} \rightarrow K$. The statement concerning the multiplication is then clear: in fact, it clearly suffices to do the case $d=l=0$, and then this results from the definition of $\Psi$. So Lemma 17 (hence also Lemma 16) is proved.

We also need the following lemma.

Lemma 18. If $\phi$ is generic, for generic $Q \in H^{0}\left({ }^{\circ}(2)\right)$, the map $\Psi(Q): K^{*} \rightarrow K$ is an isomorphism. 
Proof. Notice that each $\Psi(Q)$ is symmetric and hence defines a quadric $q_{Q}$ on $K^{*}$. In fact, $q_{Q}(k)=\chi(Q)\left(k^{2}\right)$, with the notation of the above proof. But we know that the map $\chi$ has for image the set of linear forms vanishing on $H^{0}\left(\mathbb{O}_{\mathbb{p}}(1)\right) \cdot H^{0}\left(\mathbb{O}_{X}(1)\right)$. So to prove the lemma, it suffices to show that this set, viewed as a set of quadrics on $H^{0}\left(\mathcal{O}_{X}(1)\right)$, has exactly for base locus $H^{0}\left(\mathbb{O}_{\mathbb{P}^{3}}(1)\right)$. But the base locus of this set of quadrics is exactly the set

$$
\left\{k \in H^{0}\left(\mathcal{O}_{X}(1)\right), k^{2} \in H^{0}\left(\mathcal{O}_{\mathbb{P} 3}(1)\right) \cdot H^{0}\left(\mathcal{O}_{X}(1)\right)\right\} .
$$

So we have to prove that for generic $\phi=\left(\phi_{0}, \ldots, \phi_{3}\right)$ the condition $k^{2} \in\left\langle\phi_{0}, \ldots, \phi_{3}\right\rangle$ implies that $k \in\left\langle\phi_{0}, \ldots, \phi_{3}\right\rangle$. This is easy: It suffices to degenerate $\left(\phi_{0}, \ldots, \phi_{3}\right)$ to the linear system of elements of $H^{0}\left(\mathrm{O}_{X}(1)\right)$ vanishing on a certain number of points of $X$, and verify that one can do this while keeping the dimension of $\left\langle\phi_{0}, \ldots, \phi_{3}\right\rangle \subset$ $H^{0}\left(\mathrm{O}_{X}(2)\right)$ constant. Then for the degenerated system $\left(\phi_{0}, \ldots, \phi_{3}\right)$, the result is obvious; this implies the same thing for the generic system.

Similarly, let $\lambda_{3}=\psi \phi^{*}\left(\lambda_{1}\right) \in H^{1}\left(\phi^{*} \Omega_{\Sigma}\right)_{0}$, for any $\psi \in H^{0}\left(\mathcal{O}_{X}(4)\right)$. Then the multiplication map

$$
\mu_{\lambda_{3}}: H^{0}\left(\mathcal{O}_{S}(d)\right) \longrightarrow H^{1}\left(\phi^{*} \Omega_{\Sigma}(d)\right)
$$

induces a map

$$
\bar{\mu}_{\lambda_{3}}: R_{\sigma}^{d} \cong \phi^{*}\left(H^{1}\left(T_{\Sigma}\right)\right) \longrightarrow H^{1}\left(\Omega_{\Sigma}(d-4)\right) \cong R_{\sigma}^{3 d-8},
$$

where we use Corollary 5 to realize $H^{1}\left(\Omega_{\Sigma}(d-4)\right)$ as a quotient of $H^{1}\left(\phi^{*} \Omega_{\Sigma}(d)\right)$. Then we have the following.

Lemma 19. There is a nonzero map $\Phi: H^{0}\left(\mathcal{O}_{X}(4)\right) \rightarrow \mathbb{C}$ such that $\bar{\mu}_{\lambda_{3}}$ is equal to $\Phi(\psi) \lambda_{1}$.

This is not difficult. In fact, $\Phi \in\left(H^{0}\left(\mathrm{O}_{X}(4)\right)\right)^{*}$ is simply given by the inclusion of $\mathbb{C}=H^{3}\left(\mathbb{O}_{\mathbb{P}^{3}}(-4)\right)$ in $H^{3}\left(\mathscr{O}_{X}(-4)\right)$.

Proof of Proposition 4. We know from Lemma 13 that $A \lambda_{1}:\left(\operatorname{Ker} \lambda^{\prime}\right)^{d-1} \rightarrow$ $\left(\text { Coker } \lambda^{\prime}\right)^{3 d-7}$ is an isomorphism. By Lemma 18, we also know that for generic $Q$ the map $\Psi(Q): K^{*} \rightarrow K$ is an isomorphism. Using Lemmas 14 and 16, we conclude that for generic $Q$ the map induced by $\bar{\mu}_{\lambda_{2}}$

$$
\operatorname{Ker} \bar{\mu}_{\lambda^{\prime}} \longrightarrow \operatorname{Coker} \bar{\mu}_{\lambda^{\prime}}
$$

vanishes on $\left(\operatorname{Ker} \lambda^{\prime}\right)^{d}$ and induces a (symmetric) isomorphism

$$
K^{*} \otimes\left(\operatorname{Ker} \lambda^{\prime}\right)^{d-1} \longrightarrow K \otimes\left(\operatorname{Coker} \lambda^{\prime}\right)^{3 d-7}
$$

Next by Lemma 13, we know that the map $\lambda_{1}:\left(\operatorname{Ker} \lambda^{\prime}\right)^{d} \rightarrow\left(\operatorname{Coker} \lambda^{\prime}\right)^{3 d-8}$ has for kernel exactly $\langle\bar{t}\rangle$. Using Lemmas 14 and 19 , we conclude that for generic $\psi$ the map induced by $\bar{\mu}_{\lambda_{3}}$

$$
\operatorname{Ker} \bar{\mu}_{\lambda^{\prime}} \longrightarrow \operatorname{Coker} \bar{\mu}_{\lambda^{\prime}}
$$


induces a (symmetric) map

$$
\left(\operatorname{Ker} \lambda^{\prime}\right)^{d} \longrightarrow\left(\text { Coker } \lambda^{\prime}\right)^{3 d-8}
$$

which has for kernel exactly $\langle\bar{t}\rangle$. But then it follows immediately that for generic $Q$ and $\psi$ and for $\lambda=\lambda^{\prime}+\lambda_{2}+\lambda_{3}$, the map

$$
\bar{\mu}_{\lambda}: H^{1}\left(\phi^{*}\left(T_{\Sigma}\right)\right) \longrightarrow H^{1}\left(\phi^{*} \Omega_{\Sigma}(d)\right)
$$

has its kernel generated by $\phi^{*} \bar{t} \in H^{1}\left(\phi^{*} T_{\Sigma}\right)$.

To conclude the proof of Proposition 4, we now simply note that the map

$$
\delta: H^{1}\left(\phi^{*} \Omega_{\Sigma}(d)\right) \longrightarrow H^{2}\left(\mathscr{O}_{S}\right)
$$

is injective. To see this, it suffices to prove that $H^{1}\left(\phi^{*}\left(\Omega_{\mathbb{P} 3}(d)\right)_{\mid S}\right)=0$ or that $H^{2}\left(\phi^{*}\left(\Omega_{\mathbb{p} 3}\right)\right)=0$, which is easy.

Then we have proved that $\delta \circ \bar{\mu}_{\lambda}$ has its kernel generated by $\phi^{*}(\bar{t})$ and since this map is equal to the factorization through $H^{1}\left(\phi^{*} T_{\Sigma}\right)$ of $\bar{\nabla}^{S}(\lambda): H^{0}\left(\mathcal{O}_{S}(d)\right) \rightarrow H^{2}\left(\mathbb{O}_{S}\right)$, it follows that this last map has its kernel generated by $\tilde{J}_{\Sigma}$ and $\alpha=\phi^{*} t$.

So Proposition 4 is proved.

Next we prove the following lemma.

Lemma 20. Assume that for $t$ generic in $H^{0}\left(\mathscr{O}_{\Sigma}(d)\right)$, there exists $\lambda \in H^{1}\left(\phi^{*} \Omega_{\Sigma}\right)_{0}$ such that $\bar{\nabla}^{S}(\lambda): H^{0}\left(\mathrm{O}_{S}(d)\right) \rightarrow H^{2}\left(\mathscr{O}_{S}\right)$ has its kernel generated by $\tilde{J}_{\Sigma}$ and $\alpha=$ $\phi^{*}(t)$. Then for generic $\lambda \in \operatorname{Ker} \bar{\nabla}_{\alpha}^{S} \subset H^{1}\left(\Omega_{S}\right)_{0}$ the kernel $\operatorname{Ker} \bar{\nabla}^{S}(\lambda): H^{0}\left(\mathbb{O}_{S}(d)\right) \rightarrow$ $H^{2}\left(\mathcal{O}_{S}\right)$ is generated by $\alpha$.

Proof. For any $\lambda \in H^{1}\left(\Omega_{S}\right)_{0}$, the map

$$
\bar{\nabla}^{S}(\lambda): H^{0}\left(\mathrm{O}_{S}(d)\right) \longrightarrow H^{2}\left(\mathrm{O}_{S}\right)
$$

is symmetric with respect to Serre duality, so it determines a quadric $q_{\lambda}$ on $\mathbb{P}\left(H^{0}\right.$ $\left.\left(O_{S}(d)\right)\right)$. We know by assumption that there is a $q_{\lambda}$, which has for singular locus the projective space generated by $\alpha$ and $\tilde{J}_{\Sigma}$, and we want to conclude that the generic $q_{\lambda}$ singular at $\alpha$ has $\alpha$ as its only singular point. By Bertini, it clearly suffices to prove that the system of quadrics $q_{\lambda}$ singular at $\alpha$ has no base point on the projective space $\mathbb{P}\left(\tilde{J}_{\Sigma}\right)$. Now note that the set $\operatorname{Ker} \bar{\nabla}_{\alpha}^{S}$, which exactly parametrizes this linear system, identifies to

$$
\left\{\lambda \in H^{1}\left(\Omega_{S}\right)_{0}, \lambda \perp \bar{\nabla}_{\alpha}^{S}\left(H^{0}\left(K_{S}\right)\right)\right\},
$$

where the symbols $\perp$ refer to the pairing on $H^{1}\left(\Omega_{S}\right)_{0}$. Furthermore, by definition, the condition $q_{\lambda}(u)=0$ is equivalent to $\lambda \perp \bar{\nabla}_{u}^{S}(u)$. Recalling that the map

$$
\bar{\nabla}_{u}^{S}: H^{0}\left(K_{S}\right) \longrightarrow H^{1}\left(\Omega_{S}\right)_{0}
$$


identifies to the composite of the multiplication by $u$

$$
H^{0}\left(K_{S}\right)=H^{0}\left(\mathrm{O}_{S}(d)\right) \longrightarrow H^{0}\left(\mathrm{O}_{S}(2 d)\right)
$$

and of the map (4.10)

$$
H^{0}\left(\mathrm{O}_{S}(2 d)\right) \longrightarrow H^{1}\left(\Omega_{S}\right)_{0}
$$

we conclude that $u$ is in the base locus of the system of quadrics $\operatorname{Ker} \bar{\nabla}_{\alpha}^{S}$ if and only if the image of $u^{2}$ in $H^{1}\left(\Omega_{S}\right)_{0}$ is contained in the image of $\alpha H^{0}\left(\mathscr{O}_{S}(d)\right)$ in $H^{1}\left(\Omega_{S}\right)_{0}$, which has for kernel the space $J_{S}^{2 d}$, image of $H^{0}\left(T_{X}(d)_{\mid S}\right)$ in $H^{0}\left(\mathcal{O}_{S}(d)\right)$. So the proof of Lemma 20 is concluded by the following lemma.

Lemma 21. For generic $\sigma, t$, the condition $u^{2}=\phi^{*} t . v \bmod . J_{S}^{2 d}$ for $u \in \tilde{J}_{\Sigma}^{d}, v \in$ $H^{0}\left(\mathscr{O}_{S}(d)\right)$ implies that $u=0$.

The proof of this last lemma is not very difficult, so we do not give it here.

From Lemma 20 and Proposition 4, we conclude now with the following.

Corollary 6. If for $\sigma, t$ generic, there exist $A, \lambda_{1}$ satisfying the assumptions of Proposition 4, then Proposition 3 is true.

Proof of Proposition 3. It remains only to show the existence of $A, \lambda_{1}$ satisfying conditions (a) to (e) of Proposition 4.

For any integer $k$ we have the map given by the multiplication in the Jacobian ring of $\sigma$

$$
R_{\sigma}^{2 k} \longrightarrow \operatorname{Hom}_{\text {sym }}\left(R_{\sigma}^{2 d-4-k}, R_{\sigma}^{2 d-4+k}\right),
$$

where the subscript "sym" refers to the perfect pairings (4.28). We denote by $D_{\sigma}^{2 k} \subset$ $\mathbb{P}\left(R_{\sigma}^{2 k}\right)$ the discriminant hypersurface for these families of quadrics. It is easy to show that for generic $\sigma$ and for any $0 \leq k \leq 2 d-4, D_{\sigma}^{2 k} \neq \mathbb{P}\left(R_{\sigma}^{2 k}\right)$. This is what we want to show:

For generic $\sigma$ and generic $t \in R_{\sigma}^{d}$, there exists $\lambda_{1} \in D_{\sigma}^{2 d-8}$ such that $\operatorname{Ker} \lambda_{1}$ is generated by $t$. Furthermore, for generic $A \in H^{0}\left(0_{\Sigma}(2)\right)$, we have $A^{k} \lambda_{1} \notin D_{\sigma}^{2 d-8+2 k}$, for $1 \leq k \leq 4$.

Now notice that the degree of $D_{\sigma}^{2 k}$ is equal to the rank of $R_{\sigma}^{2 d-4-k}$. In particular, we have the following:

$$
\begin{aligned}
& d^{0} D_{\sigma}^{2 d-6}=r k R_{\sigma}^{d-1}<r k R_{\sigma}^{d}=d^{0} D_{\sigma}^{2 d-8}, \\
& d^{0} D_{\sigma}^{2 d-4}=r k R_{\sigma}^{d-2}<r k R_{\sigma}^{d}=d^{0} D_{\sigma}^{2 d-8}, \\
& d^{0} D_{\sigma}^{2 d-2}=r k R_{\sigma}^{d-3}<r k R_{\sigma}^{d}=d^{0} D_{\sigma}^{2 d-8}, \\
& d^{0} D_{\sigma}^{2 d}=r k R_{\sigma}^{d-4}<r k R_{\sigma}^{d}=d^{0} D_{\sigma}^{2 d-8} .
\end{aligned}
$$

Furthermore, it is easy to show that for $\sigma$ generic and $A$ generic we have $A^{k} R_{\sigma}^{2 d-8} \not \subset$ $D_{\sigma}^{2 d-8+2 k}$ for $1 \leq k \leq 4$. 
Then we contend that the existence of $A, \lambda_{1}$ satisfying conditions (a) to (e) follows from the next lemma.

Lemma 22. Let $\sigma$ be generic, and let $\mathscr{R} \subset \mathbb{P}\left(R_{\sigma}^{d}\right) \times \mathbb{P}\left(R_{\sigma}^{2 d-8}\right)$ be defined as

$$
\mathscr{R}=\left\{\left(t, \lambda_{1}\right), t \lambda_{1}=0 \text { in } R_{\sigma}^{3 d-8}\right\} .
$$

Then $\mathscr{R}$ has only one component $\mathscr{R}_{\mathrm{gen}}$ of dimension at least equal to $\operatorname{dim} \mathbb{P}\left(R_{\sigma}^{2 d-8}\right)-1$.

Indeed, we know that for generic $t \in R_{\sigma}^{d}$, the map $t: R_{\sigma}^{2 d-8} \rightarrow R_{\sigma}^{3 d-8}$ is surjective. It follows that the principal component of $\mathscr{R}$ (the one that dominates $\mathbb{P}\left(R_{\sigma}^{d}\right)$ ) is exactly of dimension $\operatorname{dim} \mathbb{P}\left(R_{\sigma}^{2 d-8}\right)-1$, and it must be equal to $\mathscr{R}_{\text {gen }}$. So $\mathscr{R}_{\text {gen }}$ is of dimension $\operatorname{dim} \mathbb{P}\left(R_{\sigma}^{2 d-8}\right)-1$. But $\mathscr{R}_{\text {gen }}$ has to dominate $D_{\sigma}^{2 d-8}$ by the second projection, since $\mathscr{R}$ has no other component of dimension at least equal to $\operatorname{dim} D_{\sigma}^{2 d-8}$. Since $\operatorname{dim} \mathscr{R}_{\text {gen }}=\operatorname{dim} D_{\sigma}^{2 d-8}$, the second projection

$$
\mathscr{R}_{\text {gen }} \longrightarrow D_{\sigma}^{2 d-8}
$$

must be birational, and any other component of $\mathscr{R}$ is sent to a proper subset of $D_{\sigma}^{2 d-8}$. It follows that $D_{\sigma}^{2 d-8}$ is irreducible, and its generic element $\lambda_{1}$ satisfies that $\operatorname{Ker} \lambda_{1}$ is generated by $t$ for generic $t \in R_{\sigma}^{d}$. But then $D_{\sigma}^{2 d-8}$ is also reduced. For degree reasons, we cannot then have $A^{k} D_{\sigma}^{2 d-8} \subset D_{\sigma}^{2 d-8+2 k}$ for $1 \leq k \leq 4$, and since $D_{\sigma}^{2 d-8}$ is irreducible, it follows that for generic $\lambda_{1} \in D_{\sigma}^{2 d-8}$, we have $A^{k} \lambda_{1} \notin D_{\sigma}^{2 d-8+2 k}$ for $1 \leq k \leq 4$. So Lemma 22 implies the existence of $A, \lambda_{1}$ satisfying conditions (a) to (e).

Proof of Lemma 22. One has to prove that there exists no nonempty proper subset $Z \subset \mathbb{P}\left(R_{\sigma}^{d}\right)$ such that for $z \in Z$ the multiplication map $z: R_{\sigma}^{2 d-8} \rightarrow R_{\sigma}^{3 d-8}$ has cokernel of dimension at least equal to $k=\operatorname{codim} Z$. Equivalently, by duality the map $z: R_{\sigma}^{d} \rightarrow R^{2 d}$ has a kernel of dimension at least equal to $k=\operatorname{codim} Z$. Let $l \leq d$ be such that

$$
h^{0}\left(\mathcal{O}_{\mathbb{P} 3}(l)\right) \leq k<h^{0}\left(\mathcal{O}_{\mathbb{P} 3}(l+1)\right) .
$$

One first verifies that there exists $0<\epsilon<\epsilon^{\prime}<1$ such that for $d$ large enough, $\sigma$ generic, and $Z$ as above, one has $\epsilon d<l<\epsilon^{\prime} d$. This follows from the following facts, which are proved by a dimension count:

(a) there exists $0<\epsilon<1$ such that for sufficiently large $d$, generic $\sigma$, and any $t \neq 0 \in R_{\sigma}^{[\epsilon d]}$, the multiplication map $t: R_{\sigma}^{d} \rightarrow R_{\sigma}^{d+[\epsilon d]}$ is injective;

(b) there exists $B \in R_{\sigma}^{d-[\epsilon d]}$ such that the multiplication map $B: R_{\sigma}^{d+[\epsilon d]} \rightarrow R^{2 d}$ is injective.

It follows from (a) and (b) that $B R_{\sigma}^{[\epsilon]]}$ does not meet $Z$, which implies that $l+1 \geq \epsilon d$. Also it follows from (a) and (b) that for any $z \in R_{\sigma}^{d}$, we have $\operatorname{Ker} z \cap B R_{\sigma}^{[\epsilon d]}=\{0\}$. Hence for $z \in Z$, we have

$$
k \leq \operatorname{dim} \operatorname{Ker} z \leq h^{0}(d)-h^{0}([\epsilon d]) \leq h^{0}\left(\left[\epsilon^{\prime} d\right]\right),
$$


where $\epsilon^{\prime}$ is chosen so as to satisfy the last inequality for large $d$. This gives the other inequality.

Now one shows that for any $l<\epsilon^{\prime} d, d$ large enough, and for generic $\sigma$, there exists $C \in R_{\sigma}^{d-l-2}$ such that the multiplication map

$$
C: R_{\sigma}^{d+l+2} \longrightarrow R_{\sigma}^{2 d}
$$

is injective. Consider now the map $C: R_{\sigma}^{l+2} \rightarrow R_{\sigma}^{d}$. Then for $z \in R_{\sigma}^{l+2}$, we have

$$
\operatorname{Ker}\left(z: R_{\sigma}^{d} \longrightarrow R_{\sigma}^{d+l+2}\right)=\operatorname{Ker}\left(C z: R_{\sigma}^{d} \longrightarrow R_{\sigma}^{2 d}\right) ;
$$

hence, in particular, if $C z \in Z$, we have $\operatorname{dim} \operatorname{Ker}\left(z: R_{\sigma}^{d} \rightarrow R_{\sigma}^{d+l+2}\right) \geq h^{0}(l)$. Hence we conclude that if $Z^{\prime}=C R_{\sigma}^{l+2} \cap Z$, we have that the codimension of $Z^{\prime}$ in $\mathbb{P}\left(R_{\sigma}^{l+2}\right)$ is at most equal to $h^{0}(l+1)$, and for $z \in Z^{\prime}, \operatorname{dim} \operatorname{Ker}\left(z: R_{\sigma}^{d} \rightarrow R_{\sigma}^{d+l+2}\right) \geq h^{0}(l)$. This is absurd because of the following fact (which is proved by looking at the Fermat equation):

The dimension of the subspace $Z^{\prime \prime}$ of $\mathbb{P}\left(R_{\sigma}^{l+2}\right)$ defined by the condition

$$
z \in Z^{\prime \prime} \Longleftrightarrow \operatorname{dim} \operatorname{Ker}\left(z: R_{\sigma}^{d} \longrightarrow R_{\sigma}^{d+l+2}\right) \geq h^{0}(l)
$$

is not greater than 140 , for generic $\sigma$.

This obviously contradicts the fact that $Z^{\prime} \subset Z^{\prime \prime}$ and $\operatorname{dim} Z^{\prime} \geq h^{0}(l+2)-h^{0}(l+1)$, which is strictly greater than 140 for $d$ large enough, since $l>\epsilon d$.

So the existence of such $Z$ for generic $\sigma$ is absurd, and Lemma 22 is proved.

The proof of Proposition 3 is now finished, and together with Propositions 1 and 2, it implies Theorem 4.

\section{REFERENCES}

[1] A. Albano And A. Collino, On the Griffiths group of the cubic sevenfold, Math. Ann. 299 (1994), 715-726.

[2] J. CaRlson and P. Griffiths, "Infinitesimal variations of Hodge structure and the global Torelli problem" in Journées de Géométrie Algébrique (Angers, 1979), ed. A. Beauville, Sijthoff \& Noordhoff, Alphen aan den Rijn, 1980, 51-76.

[3] H. Clemens, Homological equivalence, modulo algebraic equivalence, is not finitely generated, Inst. Hautes Études Sci. Publ. Math. 58 (1983), 19-38.

[4] - "Some results about Abel-Jacobi mappings" in Topics in Transcendental Algebraic Geometry (Princeton, 1981/1982), ed. P. Griffiths, Ann. of Math. Stud. 106, Princeton Univ. Press, Princeton, 1984, 289-304.

[5] P. Deligne, Théorie de Hodge, II, Inst. Hautes Études Sci. Publ. Math. 40 (1971), 5-57.

[6] R. Donagi and E. Markman, "Cubics, integrable systems, and Calabi-Yau threefolds" in Proceedings of the Hirzebruch 65 Conference on Algebraic Geometry (Ramat Gan, 1993), Israel Math. Conf. Proc. 9, Bar-Ilan Univ., Ramat Gan, 1996, 199-221.

[7] M. GREen, The period map for hypersurface sections of high degree of an arbitrary variety, Compositio Math. 55 (1985), 135-156. 
[8] - Griffiths' infinitesimal invariant and the Abel-Jacobi map, J. Differential Geom. 29 (1989), 545-555.

[9] P. GRIFFITHS, Periods of integrals on algebraic manifolds, I, Amer. J. Math. 90 (1968), 568-626; II, 805-865.

[10] - On the periods of certain rational integrals, I, Ann. of Math. (2) 90 (1969), 460-495; II, 496-541.

[11] - Infinitesimal variations of Hodge structure, III: Determinantal varieties and the infinitesimal invariant of normal functions, Compositio Math. 50 (1983), 267-324.

[12] S.-O. KIM, Noether-Lefschetz locus for surfaces, Trans. Amer. Math. Soc. 324 (1991), 369-384.

[13] M. NoRI, Algebraic cycles and Hodge-theoretic connectivity, Invent. Math. 111 (1993), 349373.

[14] - Cycles on the generic abelian threefold, Proc. Indian Acad. Sci. Math. Sci. 99 (1989), 191-196.

[15] K. Paranjape, Curves on threefolds with trivial canonical bundle, Proc. Indian Acad. Sci. Math. Sci. 101 (1991), 199-213.

[16] Z. RAN, Hodge theory and the Hilbert scheme, J. Differential Geom. 37 (1993), 191-198.

[17] C. Voisin, Densité du lieu de Noether-Lefschetz, pour les sections hyperplanes des variétés de Calabi-Yau de dimension 3, Internat. J. Math. 3 (1992), 699-715.

[18] - Sur l'application d'Abel-Jacobi des variétés de Calabi-Yau de dimension trois, Ann. Sci. École Norm. Sup. (4) 27 (1994), 209-226.

Institut de Mathématiques de Jussieu, Centre National de la Recherche Scientifique, Unité Mixte de Recherche 9994, 75251 Paris Cedex 05, France 